\title{
Cooperative gene activation by AF4 and DOT1L drives MLL-rearranged leukemia
}

\author{
Hiroshi Okuda, ${ }^{1}$ Boban Stanojevic,, ${ }^{1,2}$ Akinori Kanai, ${ }^{3}$ Takeshi Kawamura, ${ }^{4}$ Satoshi Takahashi, ${ }^{5}$ Hirotaka Matsui, ${ }^{3,6}$ \\ Akifumi Takaori-Kondo, ${ }^{5}$ and Akihiko Yokoyama ${ }^{1,5,7,8}$

\begin{abstract}
'Laboratory for Malignancy Control Research, Kyoto University Graduate School of Medicine, Kyoto, Japan. ²Laboratory for Radiobiology and Molecular Cenetics, Vinča Institute of Nuclear Sciences, ${ }^{4}$ Research Center for Advanced Science and Technology (RCAST), The University of Tokyo, Tokyo, Japan. ${ }^{5}$ Department of Hematology and Oncology, Kyoto University Graduate School of Medicine, Kyoto, Japan. ${ }^{6}$ Department of Molecular Laboratory Medicine, Graduate School of Medical Sciences, Kumamoto University, Kumamoto, Japan. ${ }^{7}$ Division of Hematological Malignancy, National Cancer Center Research Institute, Tokyo, Japan. ${ }^{8}$ Tsuruoka Metabolomics Laboratory, National Cancer Center, Yamagata, Japan.
\end{abstract} \\ University of Belgrade, Belgrade, Serbia. ${ }^{3}$ Department of Molecular Oncology and Leukemia Program Project, Research Institute for Radiation Biology and Medicine, Hiroshima University, Hiroshima, Japan.
}

\begin{abstract}
The eleven-nineteen leukemia (ENL) protein family, composed of ENL and AF9, is a common component of 3 transcriptional modulators: AF4-ENL-P-TEFb complex (AEP), DOT1L-AF10-ENL complex (referred to as the DOT1L complex) and polycombrepressive complex 1 (PRC1). Each complex associates with chromatin via distinct mechanisms, conferring different transcriptional properties including activation, maintenance, and repression. The mixed-lineage leukemia (MLL) gene often fuses with ENL and AF10 family genes in leukemia. However, the functional interrelationship among those 3 complexes in leukemic transformation remains largely elusive. Here, we have shown that MLL-ENL and MLL-AF10 constitutively activate transcription by aberrantly inducing both AEP-dependent transcriptional activation and DOT1L-dependent transcriptional maintenance, mostly in the absence of PRC1, to fully transform hematopoietic progenitors. These results reveal a cooperative transcriptional activation mechanism of AEP and DOT1L and suggest a molecular rationale for the simultaneous inhibition of the MLL fusion-AF4 complex and DOT1L for more effective treatment of MLL-rearranged leukemia.
\end{abstract}

\section{Introduction}

Deregulation of gene expression can cause devastating cancers. Fusion of the mixed-lineage leukemia $(M L L)$ gene (also known as KMT2A, MLL1, ALL-1, or HTRX) and various partners causes aggressive leukemia (1). The resultant MLL fusion proteins constitutively activate their target genes, such as homeobox A9 (HOXA9) and Meis homeobox 1 (MEIS1), whose expression levels are elevated in the hematopoietic stem cell/multipotent progenitor fractions and are progressively suppressed during differentiation $(2,3)$. MLL fusion proteins form a complex with MENIN and lens epithelium-derived growth factor (LEDGF, also known as PSIP1) to recognize their target genes $(4,5)$ by binding di- or trimethylated histone $\mathrm{H} 3$ lysine 36 (H3K36me2/3) through the PWWP domain of LEDGF and nonmethylated CpGs through the CXXC domain of MLL (6-9). Consequently, the target chromatin of MLL is mostly located at the promoter proximal region, where CpGs are enriched (6).

To date, more than 70 genes have been reported to fuse with $M L L$ (10). Despite its promiscuity, the majority of $M L L$-rearranged leukemia ( $M L L$-r leukemia) cases are caused by MLL fusion with a component of either the AF4-ENL-P-TEFb (AEP) complex or the DOT1L-AF10-ENL complex (referred to as the DOT1L complex)

Authorship note: H. Okuda and B. Stanojevic contributed equally to this work. Conflict of interest: A. Yokoyama was supported by research funding from Dainippon Sumitomo Pharma Co., Ltd.

Submitted: October 24, 2016; Accepted: February 16, 2017.

Reference information: / Clin Invest. 2017;127(5):1918-1931.

https://doi.org/10.1172/JCI91406.
(11-16). These 2 complexes include a member of the ENL family such as eleven-nineteen leukemia (ENL, also known as MLLT1) and AF9 (also known as MLLT3). The ENL family proteins contain a YEATS domain, which recognizes acetylated histone $\mathrm{H} 3$ lysine 9, 18, or 27 (H3K9/18/27ac) (17). AEP also contains AF4 family proteins, including AF4 (also known as AFF1) and AF5Q31 (also known as AFF4), which provide various interaction platforms for cofactors (11, 14-16). The AF4 family proteins associate with the phosphorylated transcription elongation factor $\mathrm{b}$ (P-TEFb) complex and the ELL protein family, both of which demonstrate transcription elongation activities $(18,19)$. Additionally, AF4 family proteins associate with selectivity factor 1 (SL1), containing TATA box-binding protein (TBP) and TBP-associated factor RNA polymerase I subunits A through D (TAF1A/B/C/D), to initiate RNA polymerase II-dependent (RNAP2-dependent) transcription, which is the critical step activated by MLL-AEP fusion proteins in leukemic transformation $(20,21)$. Thus, AEP is a multifunctional coactivator that can facilitate both the initiation and elongation of transcription.

DOT1L is an epigenetic modifier that produces mono-, di-, and trimethylated histone H3 lysine 79 (H3K79me1/2/3) (22, 23) and maintains a protective chromatin environment against sirtuin 1-dependent (SIRT1-dependent) gene silencing (24). The DOT1L complex is mainly composed of DOT1L, an ENL family protein, and an AF10 family protein, such as AF10 (also known as MLLT10) or AF17 (also known as MLLT6) (13). The association of DOT1L with AF10 increases the histone methyltransferase (HMT) activity of DOT1L to produce highly methylated H3K79me2/3 markers (25), and their presence, in turn, correlates with active transcription. MLL-AF10 transforms hematopoietic progenitors 
through the DOT1L interaction domain (26), while MLL-ENL/ AF9 does so through the ANC1 homology domain (AHD), which is responsible for its association with both AF4 and DOT1L (11, $12,27)$. Genetic ablation of Dot1l results in the loss of clonogenic activity of MLL-AF10- and MLL-AF9-transformed hematopoietic progenitors, indicating that the presence of DOT1L is required for MLL fusion-dependent leukemic transformation (28-32). Selective inhibitors of DOT1L HMT that show efficacy against $M L L-\mathrm{r}$ leukemia have been developed $(33,34)$. Paradoxically, ENL family proteins associate with polycomb-repressive complex 1 (PRC1), which is known to suppress transcription (12). Upon double-strand breaks, ATM (ataxia telangiectasia mutated) phosphorylates the ENL family protein to switch its binding partner from the AF4 family protein to PRC1 to suppress transcription (35). However, the interrelationship among AEP, the DOT1L complex, and PRC1 and its implications in MLL fusion-dependent leukemogenesis are largely unclear.

In this study, we investigated the mechanisms of gene regulation by the 3 ENL-containing complexes and identified 2 essential transcriptional properties that are overactivated by MLL-ENL and MLL-AF10 in leukemogenesis, both of which should be simultaneously targeted to efficiently eradicate MLL stem cells.

\section{Results}

ENL colocalizes with components of the AEP and DOT1L complexes in the promoter proximal coding regions. To dissect the biochemical properties of ENL bound to chromatin, we performed immunoaffinity purification from subcellular fractions containing nucleosomes (nucleosome fraction immunoprecipitation [nucfrIP]) (6) (Figure 1A and Supplemental Figure 1, A and B; supplemental material available online with this article; https://doi.org/10.1172/ JCI91406DS1). Consistent with a previous report (12), we found that ENL associated with components of AEP (e.g., AF5Q31 and cyclin T1), the DOT1L complex (e.g., DOT1L and AF17), and PRC1 (e.g., chromobox 2 [CBX2] and BMI1 proto-oncogene, polycomb ring finger [BMI1]) via its $\mathrm{AHD}$, as shown by the failure of an AHD-lacking ENL mutant to coprecipitate with these cofactors. A similar coprecipitation analysis in more stringent conditions (fractionation-assisted native ChIP [fanChIP]), followed by Western blotting (WB) (6), showed that MLL-ENL associated with these components of the AEP, DOT1L, or PRC1 complexes (Figure 1B). ENL interacts with the 3 distinct complexes in a mutually exclusive manner (11, 36-38). Thus, exogenously expressed CBX8, a PRC1 component, pulled down ENL and BMI1, but not AEP- or DOT1L complex-specific components (Figure 1C). Mass spectrometric (MS) analysis of the nucleosomes copurified with ENL showed enrichment of both unmodified and acetylated lysine 27 on histone H3 (unmodified H3K27 and H3K27ac, respectively) (Figure 1D), consistent with the model demonstrating that the YEATS domain of ENL specifically associates with H3K27ac (17) and the PZP domain of AF10 family proteins binds to unmodified H3K27 (39).

To examine the genomic localization of these 3 complexes, we used 293T cells to perform ChIP, followed by deep sequencing (ChIP-seq). The ChIP signals of ENL, AF4, TAF1C, DOT1L, and AF17 were highly enriched at ENL target genes such as ribosomal protein L13a (RPL13A) and sorting nexin 5 (SNX5) (Figure $1 \mathrm{E})$. These AEP, SL1, and DOT1L complex components were also observed at known AEP target genes such as HOXA9 (11) (Supplemental Figure $1 C$ ), which was confirmed by a more sensitive ChIP-quantitative PCR (ChIP-qPCR) analysis (Supplemental Figure 1D). The distribution pattern of ENL relative to the transcriptional start sites (TSSs) indicated that ENL was enriched within the promoter proximal coding region 0 to $+2 \mathrm{~kb}$ from the TSSs, colocalizing with H3K9/27ac (Figure 1F). Components of AEP, SL1, and the DOT1L complex were also enriched at the promoter proximal coding region. In contrast, BMI1 and CBX8 were not specifically localized to these ENL target genes, which were actively transcribed, as indicated by the ChIP signal of RNAP2 and the RNA-seq profile (Figure 1, E and F, and Supplemental Figure 1, C and D). Taken together, AEP and the DOT1L complex act at the promoter proximal region where PRC1 is absent, suggesting that AEP and the DOT1L complex may cooperate to activate gene expression in the absence of PRC1.

DOT1L is required for AEP-SL1 complex-dependent gene activation. Given the proximity of the localization of AEP and the DOT1L complex, we hypothesized that DOT1L plays an active role in AEP-dependent transcriptional activation. To examine the role of DOT1L in the expression of AEP-SL1 target genes, we knocked down Dot 1 in immortalized mouse embryonic fibroblasts (iMEFs) using shRNA. The expression of homeobox C8 (Hoxc8), homeobox C9 (Hoxc9), and cyclin-dependent kinase inhibitor 2C (Cdkn2c) depends on Enl and Taflc in iMEFs (Figure 2A) (20). Knockdown of Dot 1 l with 2 different shRNAs decreased the expression of these AEP-SL1 target genes. RNA-seq analysis showed that Dot 11 knockdown globally reduced the expression of the AEP-SL1 target gene set (Figure 2B), which was defined as the genes commonly downregulated by knockdown of Enl and Taflc (Supplemental Figure $2 \mathrm{~A})$. These results indicate that the DOT1L complex is required for efficient AEP-SL1-dependent gene activation under non- $M L L-r$ conditions. Such cooperative gene activation was also observed in a hematopoietic context, as AEP-SL1 components colocalized with the DOT1L complex at the MYC locus in K562 leukemia cells (Supplemental Figure 2B), and knockdown of each component resulted in a decrease in $M Y C$ expression (Supplemental Figure 2C). These results suggest that AEP-DOT1L collaboration is common among various cellular contexts.

The MLL-ENL-AEP hybrid complex, the DOT1L complex, and $P R C 1$ differentially localize on chromatin. To determine the roles of AEP, the DOT1L complex, and PRC1 in MLL-ENL-dependent gene activation in leukemia cells, we analyzed the genomic localization of each component in HB1119 leukemia cells, which endogenously express both MLL-ENL and WT MLL (40). ChIP-seq analysis using 2 different antibodies showed that MLL proteins localized mainly to the region -1 to $+2 \mathrm{~kb}$ from the TSS (Figure 3, A and B, and Supplemental Figure 3A), a finding that was supported by ChIP-qPCR analysis on select loci (Supplemental Figure 3B). Since near-complete knockdown of WT MLL by shRNA did not compromise the expression of HOXA9 or MEIS1 or the recruitment of MLL proteins and MLL-ENL-specific cofactors (Supplemental Figure 3, C-F), the ChIP signal of MLL proteins can be attributed mostly to MLL-ENL.

Distribution patterns of AF4 and TAF1C were nearly identical to that of MLL-ENL (Figure 3, A and B). The ChIP signal intensity of MLL-ENL was highly correlated with that of AF4 $(r=0.8398)$ 

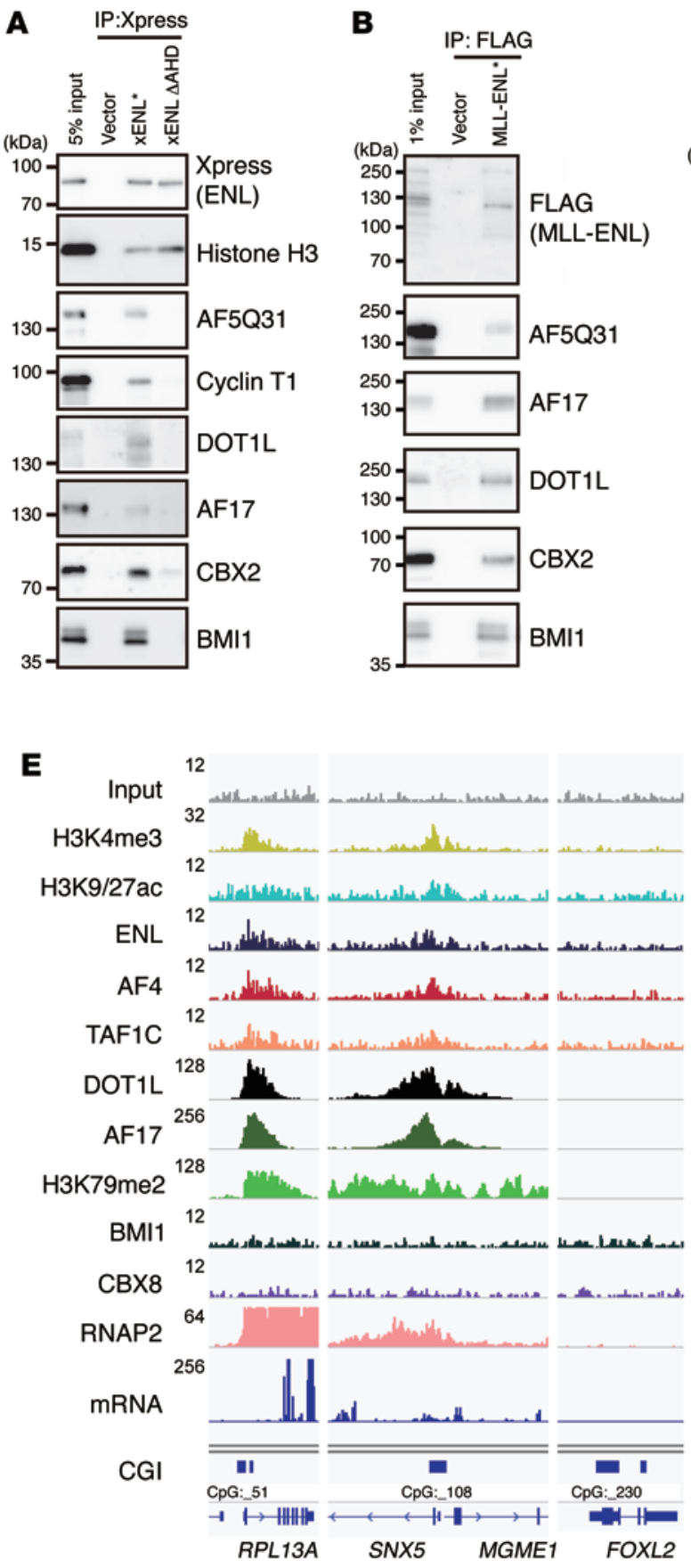

C

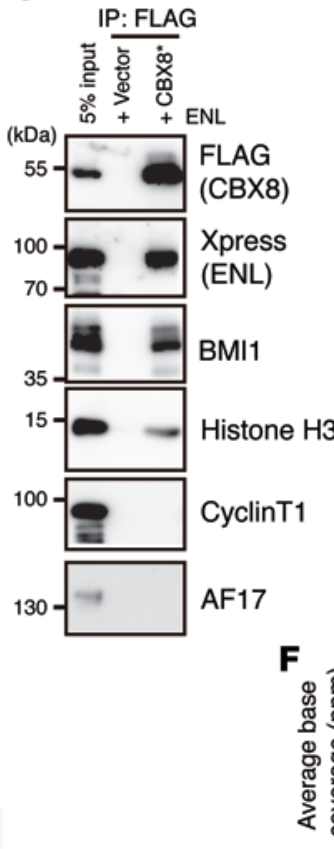

D
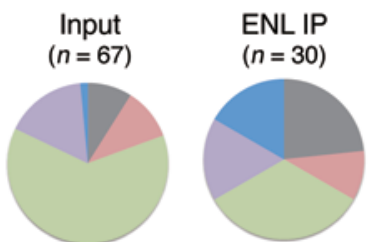

H3K27

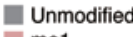

me1

me2

me3

ac

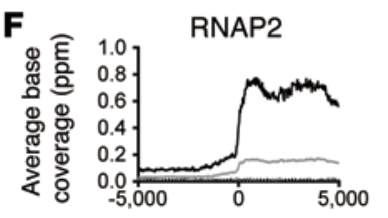

- ChIP (all genes)

Input (all genes)

- ChIP (ENL target genes)

.... Input (ENL target genes)
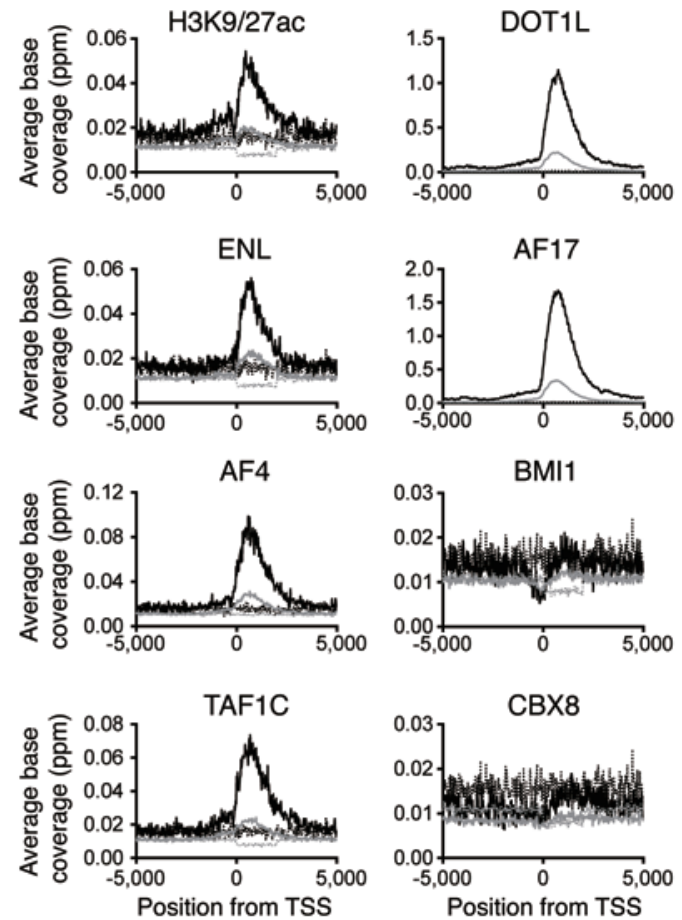

Figure 1. Genomic localization of 3 ENL-containing complexes. (A) ENL-associating factors on nucleosomes. 293T cells transiently expressing Xpresstagged ENL ( $x E N L)$ or its AHD-lacking mutant $(\triangle A H D)$ were analyzed by nucfrlP copurification using an anti-Xpress antibody. The sample shown in the input lane is indicated by an asterisk. (B) Cofactor binding of MLL-ENL. 293T cells transiently expressing FLAG-tagged MLL-ENL were analyzed by fanChIP copurification using an anti-FLAC antibody. (C) CBX8-associating factors on nucleosomes. 293T cells transiently expressing Xpress-tagged ENL, with or without FLAG-tagged CBX8, were analyzed by nucfrIP copurification using an anti-FLAG antibody. (D) Histone H3K27 modification of the nucleosomes coprecipitated with ENL. Purified nucleosomes, as in A, were subjected to MS analysis. The ratio of each modification identified by MS on histone H3K27 is shown in pie charts. The $n$ values shown indicate the number of peptides analyzed. ac, acetylated. (E) Genomic localization of ENL-containing complexes and various histone modifications in 293T cells. fanChIP or faxChIP (for H3K79me2), followed by deep sequencing, was performed on the chromatin of $293 \mathrm{~T}$ cells for the indicated proteins and modifications. ChIP signals were visualized using the Integrative Genomics Viewer (The Broad Institute). The minimum value of the $y$ axis is set at 0 , while the maximum value for each sample is indicated. The RNA-seq profile is also shown for comparison. CGI, CpC island; FOXL2, forkhead box L2; MGME1, mitochondrial genome maintenance exonuclease 1. (F) Distribution patterns of various proteins and modifications at the ENL target loci. A set of genes (852 genes) whose ENL ChIP signal within the region 0 to $+2 \mathrm{~kb}$ from the TSS was more than 2 -fold of the input signal was defined as the representative ENL target set. The frequency of each protein and modification at the ENL target TSSs (black lines) or all TSSs (gray lines) is shown along with the input DNA (dashed lines). The $y$ axis indicates the frequency of the ChIP-seq tag count (ppm) in 25-bp increments. 
A

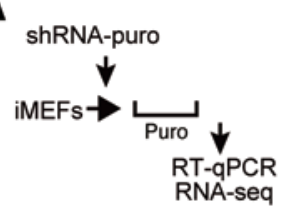

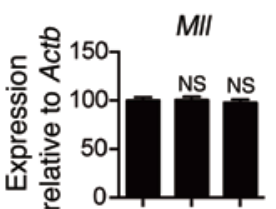

Enl
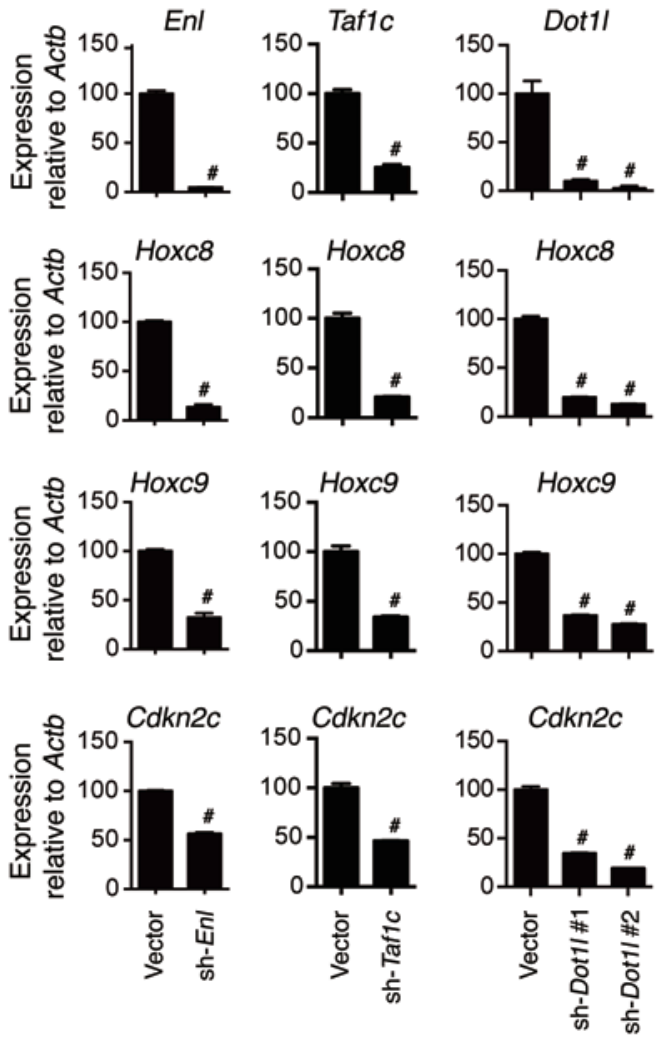

B
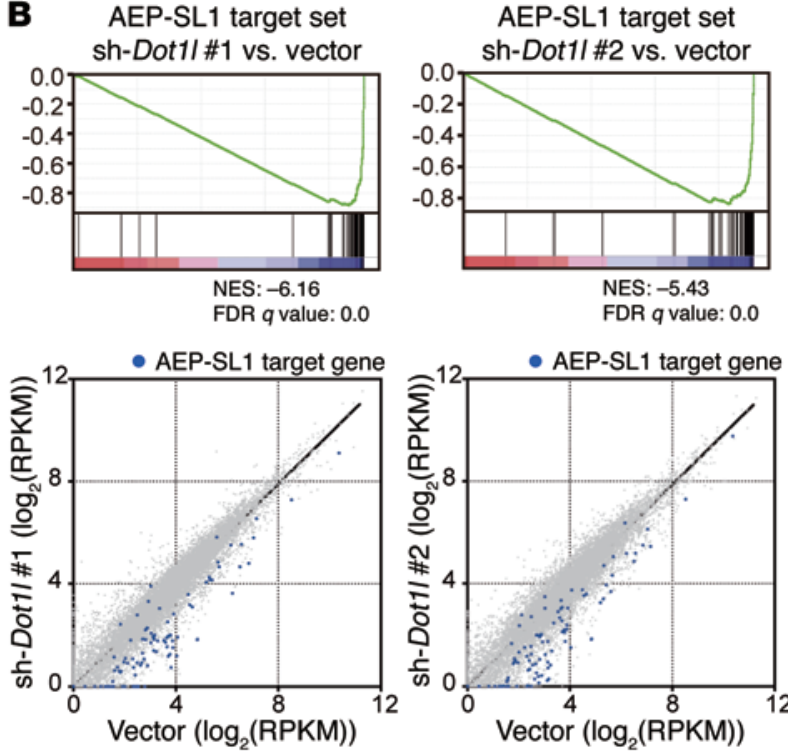

AEP-SL1 target set

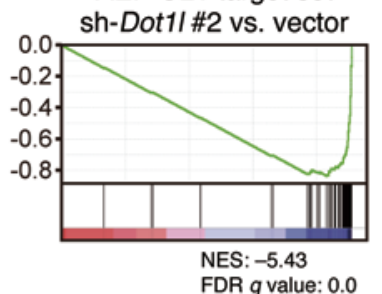

AEP-SL1 target gene

Figure 2. Role of DOT1L in AEP-dependent gene activation. (A) RT-qPCR analysis of AEP-SL1 target genes after knockdown of Dot1l in iMEFs. Data for Enl or Taf1c knockdown are included for comparison. Expression levels normalized to Actb (representative of 2 independent experiments) are shown as the value relative to that of the vector control (set at $100 \%$ ). Error bars represent the SD of PCRs performed in triplicate. ${ }^{\# P} \leq 0.0001$, by unpaired, 2 -tailed $t$ test (for sh-Enl or Taf1c) or ordinary 1-way ANOVA (for sh-Dot1l) comparing each sample with the vector control. puro, puromycin. (B) RNA-seq analysis after knockdown of Dot1l in iMEFs. The AEP-SL1 target gene set was defined as the genes whose expression levels were reduced by more than 3-fold by shRNAs for both EnI and Taf1c. GSEA and scatter plots are shown. AEP-SL1 target genes are highlighted in blue. The normalized enrichment score (NES) and FDR are indicated.

and TAF1C ( $r=0.8689)$ (Figure 3C and Supplemental Figure 3G), suggesting that AEP and SL1 are tethered to chromatin by MLLENL. The distribution patterns of DOT1L and AF17 were similar to those of AF4 and TAF1C, but skewed toward coding regions (Figure 3B). Moreover, the ChIP signal of DOT1L complex components was not in proportion to that of MLL-ENL at some loci (Figure 3A, NR3C1 and BCL7A). Consequently, localization of DOT1L $(r=0.7096)$ and $\mathrm{AF} 17(r=0.7154)$ was loosely correlated with that of MLL-ENL, compared with localization of AEP-SL1 components (Figure 3C and Supplemental Figure 3G). These results indicate that MLL-ENL primarily associates with AEP components on chromatin, while chromatin association with the DOT1L complex is partly dependent on the chromatin context in addition to the presence of MLL-ENL.

Most of the MLL target chromatin retains a profile of transcriptionally active chromatin with the ChIP signals of di- or trimethylated histone $\mathrm{H} 3$ lysine 4 (H3K4me2/3), H3K27ac, H3K79me2, and RNAP2 (Figure 3, A and B and Supplemental Figure 3, A and B). Trimethylated histone $\mathrm{H} 3$ lysine 27 (H3K27me3) was depleted from the MLL target chromatin, with the exception of the HOXA9 locus, which may be heterochromatinized in a monoallelic manner. BMI1 and CBX8 were absent from most of the MLL target promoters, though they were present in a subset of MLL target loci such as fat storage-inducing transmembrane protein 2 (FITM2) and insulin-like growth factor 1 receptor (IGF1R). Distribution patterns of BMI1 and CBX8 were skewed toward upstream noncoding regions. Most of the MLL target genes that were enriched with BMI1 and CBX8 were genes with relatively low levels of MLL-ENL, $\mathrm{AF} 4$, and DOT1L localization (Figure 3C and Supplemental Figure $3 G)$. These results indicate that MLL-ENL primarily forms a MLLENL-AEP hybrid complex at the target promoter in the absence of PRC1 and frequently coexists with DOT1L in its proximal coding region, while PRC1 colocalizes with MLL-ENL at the upstream noncoding region of a subset of MLL target genes, where AEP and DOT1L complexes are scarce.

DOT1L recruitment to the $M L L$ target loci delays gene silencing. AF10 associates with DOT1L through the octapeptide motif and leucine zipper domain (OM-LZ) (26), and AF5Q31 associates with AF4 through the carboxyl-terminal homology domain (CHD), while ENL binds to both DOT1L and AF4 via AHD (Figure 4A) (11). To determine the role of each cofactor in transcriptional activation, we generated GAL4 fusion constructs for each binding platform and performed a transactivation assay using 293TL cells (20), in which the galactose-responsive transcription factor GAL4-responsive (GAL4-responsive) reporter was integrated into the genome. The GAL4-AHD fusion protein (GAL4-ENL') exhibited substantial transactivation activity ( 13 -fold increase), while the GAL4-CHD fusion protein (GAL4-AF5-4) showed 


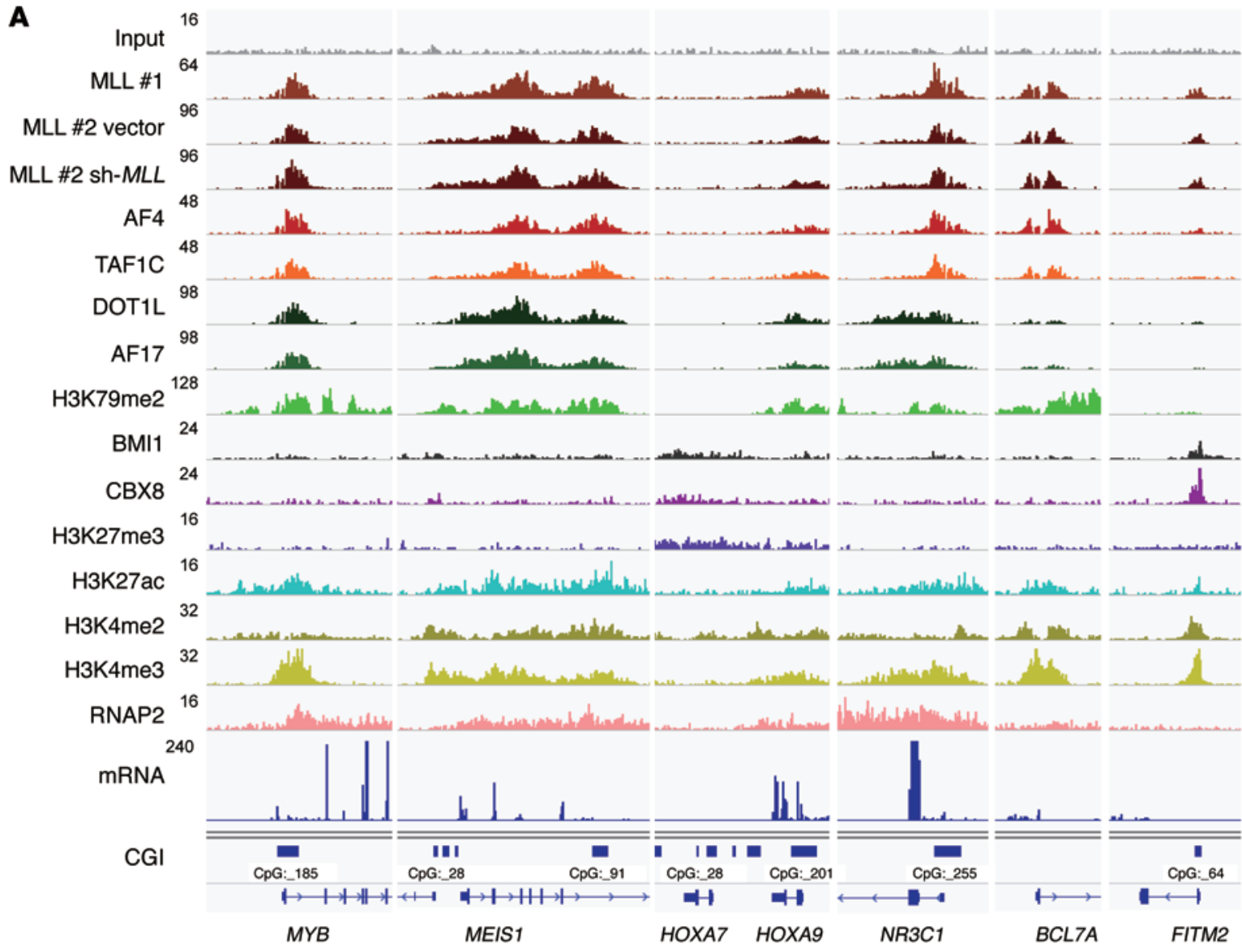

B

- ChIP (MLL target genes)

- Input (MLL target genes)

- ChIP (all genes)

- Input (all genes)
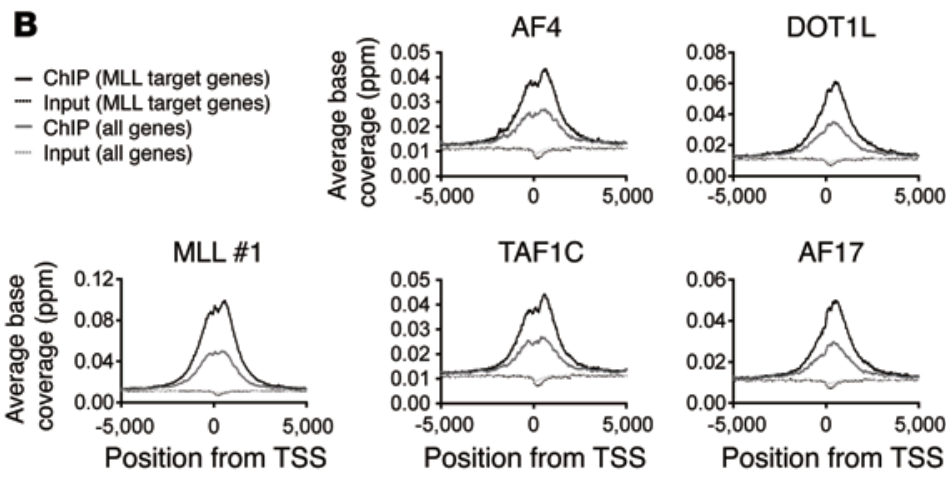

TAF1C
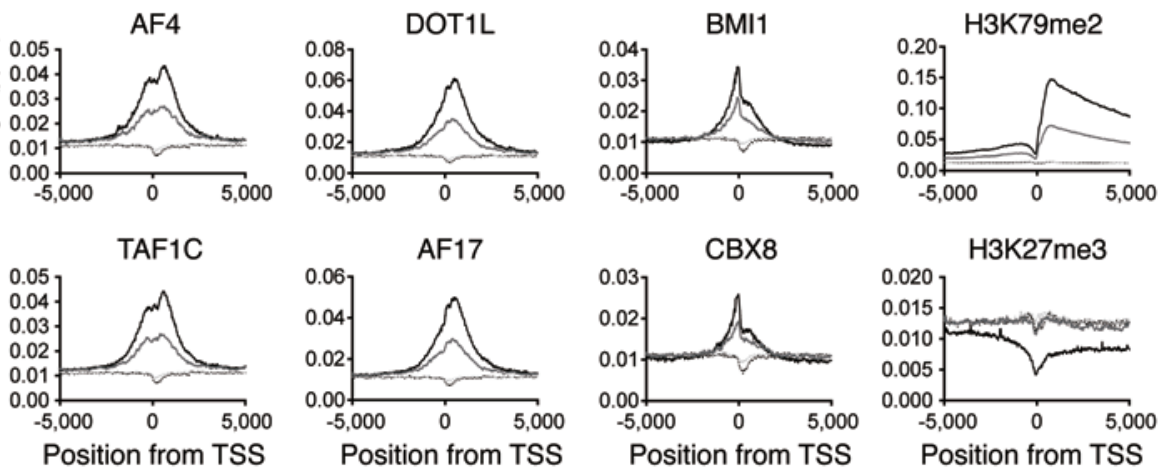

MLL vs. CBX8 (-)

C MLL vs. AF4

MLL vs. DOT1L
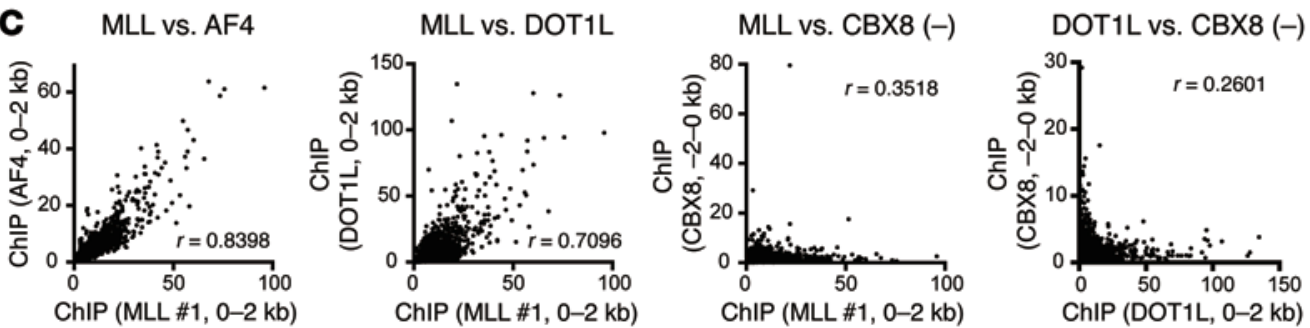

ChIP (MLL \#1, 0-2 kb)

AF4 vs. CBX8 (-)

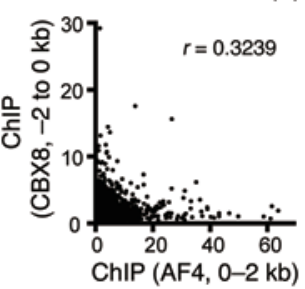

Figure 3. Genomic landscape of MLL-ENL target chromatin. (A) Genomic localization of various proteins/modifications in HB1119 cells. fanChIP or faxChIP (for H3K79me2 and H3K27me3), followed by deep sequencing, was performed on the chromatin of HB1119 cells by using specific antibodies against the indicated proteins. ChIP-seq data for RNAP2 were redundant with a previously published data set (20). RNA-seq profile is included for comparison. BCL7A, $\mathrm{BCL}$ tumor-suppressor 7A; MYB, MYB proto-oncogene, transcription factor; NR3C1, nuclear receptor subfamily 3 group $C$ member 1 . (B) Distribution patterns of various proteins/modifications at the MLL target loci. The distribution pattern relative to the TSS was analyzed as described in Figure 1F. A set of genes ( 8,830 genes) whose MLL ChIP signal within the region 0 to $+2 \mathrm{~kb}$ from the TSSs was greater than 3 -fold that of the input was defined as the representative MLL target set. (C) Relative occupation of various factors at MLL target genes. ChIP-seq tags of the MLL target set were clustered into a 2-kb bin (either 0 to $+2 \mathrm{~kb}$ from the TSS or -2 to $0 \mathrm{~kb}$, denoted as "(-)") and are presented as XY scatter plots. Spearman's rank correlation coefficient ( $r$ ) is shown. 
A

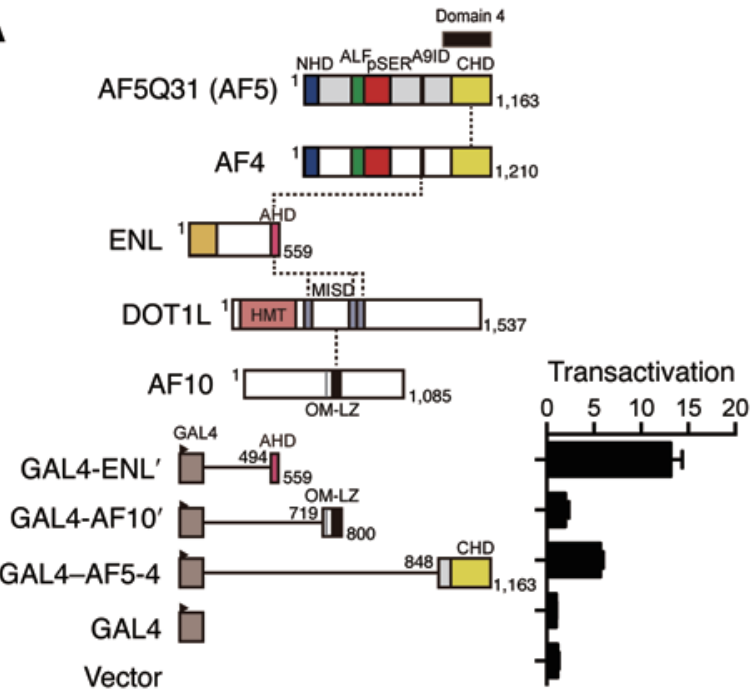

B

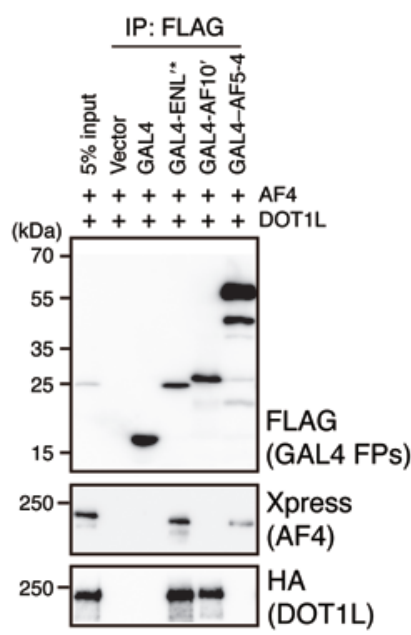

C UAS $\times 5$

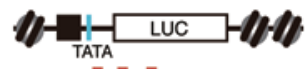

员点

Pre-TSS TSS POS-TS
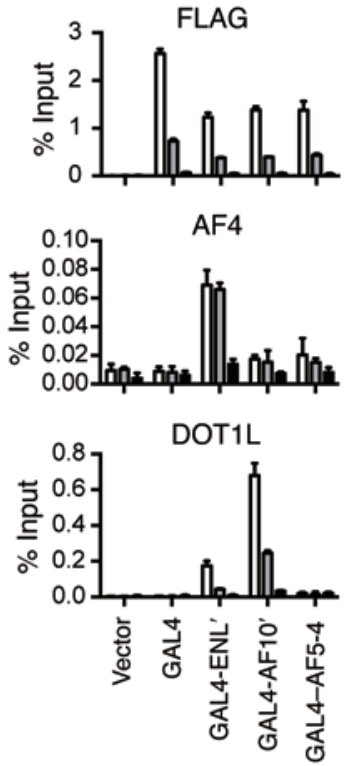
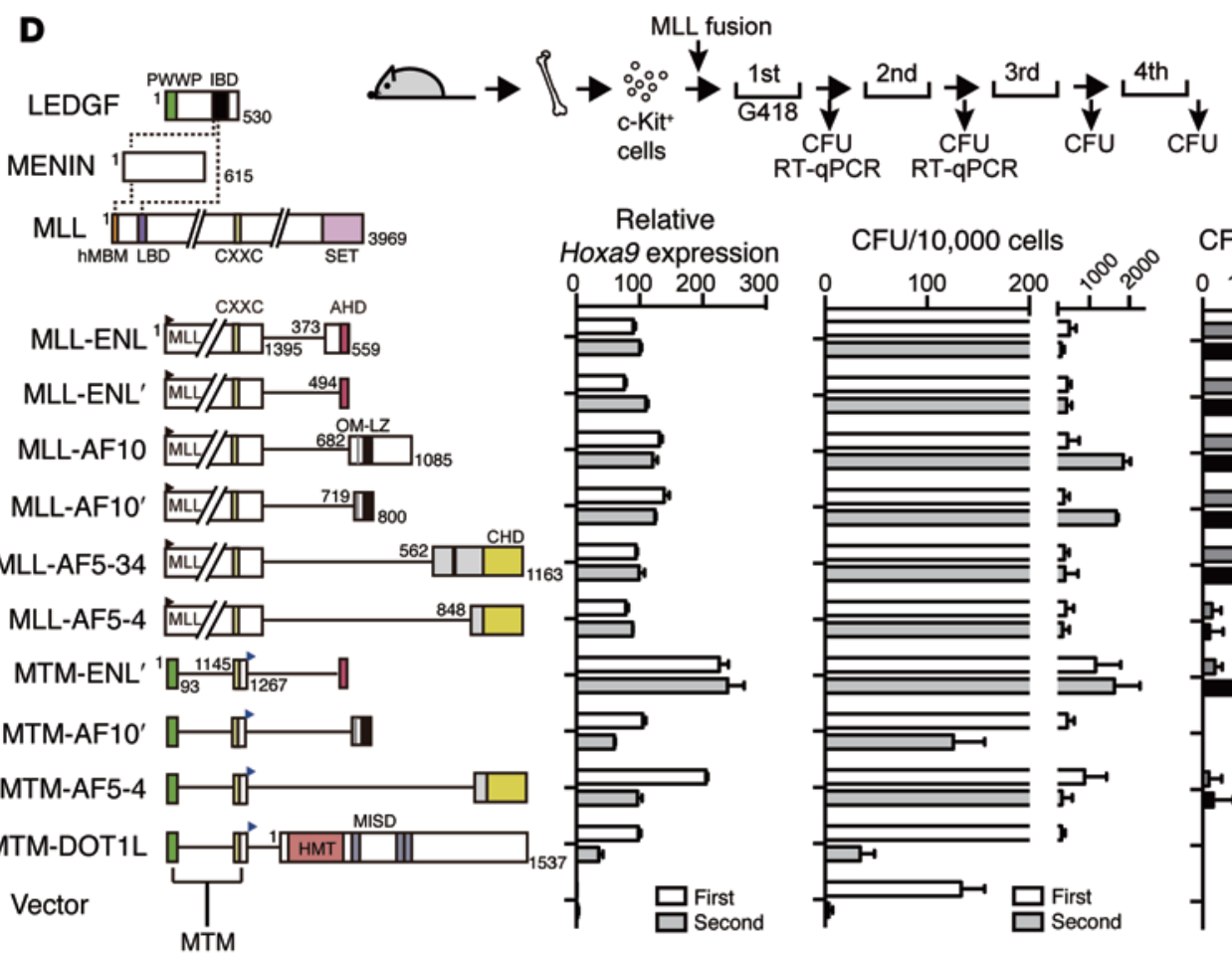

CFU/10,000 cells

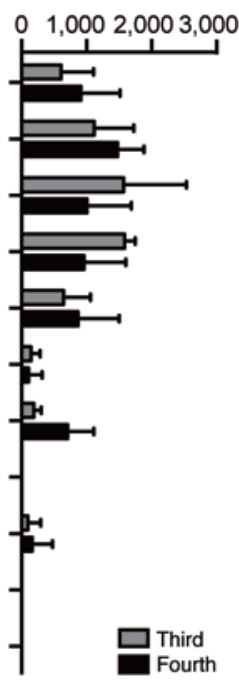

Figure 4. DOT1L delays gene silencing induced by differentiation. (A) Transactivation activities of various binding platforms. Schematic structures of various FLAG-tagged GAL4 fusion proteins are shown. Transactivation activity normalized to Renilla luciferase activity is shown (error bars represent the SD of triplicate experiments) as the value relative to that of GAL4 (set at 1). Black flag denotes the FLAG epitope. NHD, N-terminal-conserved domain; ALF, AF4-LAF4-FMR2 homology domain; pSER, poly-serine; A9ID, AF9 interaction domain; MISD, minimum interaction site of DOT1L. (B) Association of AF4 and DOT1L with various binding platforms. FLAG-tagged GAL4 fusion proteins were coexpressed with Xpress-tagged AF4 and HA-tagged DOT1L and analyzed by fanChIP-WB using an anti-FLAG antibody. Coprecipitated proteins were visualized using specific antibodies against the indicated tags. FPs, fusion proteins. (C) Recruitment of cofactors by various binding platforms. Each FLAG-tagged GAL4 fusion protein was expressed in $293 \mathrm{TL}$ cells and analyzed by ChIP-qPCR using the indicated antibodies and probes specific for the pre-TSS, TSS, and post-TSS regions of the GAL4-responsive luciferase reporter. ChIP signals are expressed as the percentage of input. Error bars represent the SD of PCRs performed in triplicate. UAS, upstream activation sequence; LUC, Luciferase reporter gene; TATA, TATA element. (D) Transforming ability of various MLL fusion proteins. The schema depicts the myeloid progenitor transformation assay. RT-qPCR was performed on first- and second-round colonies. The Hoxa9 expression level normalized to Gapdh (representative of 2 independent experiments) is shown as the value relative to that of MLL-ENL in the second-round colonies (set at $100 \%$ ). Error bars represent the SD of PCRs performed in triplicate. The number of CFU of each round is shown with error bars (SD from $>3$ independent experiments). Blue flag denotes the HA epitope. CXXC, CXXC domain; hMBM, high-affinity MENIN-binding motif; IBD, integrase-binding domain; LBD, ligand-binding domain; PWWP, PWWP domain; SET, SET domain. 
relatively weak transactivation activity ( $\sim 6$-fold increase). The GAL4-OM-LZ fusion protein (GAL4-AF10') slightly activated transcription ( 2-fold increase). Specific association with each binding platform was confirmed by fanChIP-WB (Figure $4 \mathrm{~B}$ ). ChIP-qPCR analysis showed that each binding platform recruited its specific binding partners to the GAL4-responsive elements (Figure 4C and Supplemental Figure 4A). It should be noted that GAL4-AF10' induced AF4 recruitment to a small extent, suggestive of a role of DOT1L as an AEP recruiter. The degree of AF4 recruitment roughly corresponded to the degree of transactivation (Figure 4, A and C). Both DOT1L and AEP-SL1 complexes were recruited by GAL4-ENL' (Figure 4C and Supplemental Figure $4 \mathrm{~B})$. In a condition in which DOT1L knockdown resulted in a decrease of HOXA9 expression (Supplemental Figure 4, C and D), transactivation activity of GAL4-ENL' was not impaired (Supplemental Figure 4E). These results indicate that AEP, not the DOT1L complex, is largely responsible for transcriptional activation, while the DOT1L complex may play a role in recruiting/ tethering AEP to the target chromatin.

We previously identified the minimum targeting module (MTM) necessary and sufficient for target chromatin recognition by MLL fusion proteins as the PWWP domain of LEDGF and the CXXC domain of MLL (6). To examine the functions of these binding platforms in the context of MLL fusion in hematopoietic cells, we generated constructs in which the 5 ' portion of MLL or MTM was fused to each binding platform and performed a myeloid progenitor transformation assay (Figure 4D and Supplemental Figure 4F). In this assay, functional MLL fusion proteins constitutively activate Hoxa9 to immortalize myeloid progenitors. The MLL-AHD (MLLENL') and MLL-OM-LZ (MLL-AF10') fusion proteins activated Hoxa 9 and immortalized myeloid progenitors in a manner similar to that seen with naturally occurring MLL fusion proteins (MLLENL and MLL-AF10) in clinical cases. The MLL-CHD fusion protein (MLL-AF5-4) also activated Hoxa9 and immortalized myeloid progenitors, although it was the weakest in terms of clonogenicity. The MTM-AHD fusion protein (MTM-ENL') successfully transformed myeloid progenitors, while the MTM-CHD fusion protein (MTM-AF5-4) immortalized the cells at low clonogenicity, similar to that observed with MLL-AF5-4. Myeloid progenitors transduced with the MTM-OM-LZ fusion protein (MTM-AF10') maintained a high level of Hoxa9 expression in the first-round colonies and progressively lost this expression and clonogenicity in later rounds, unlike MLL-AF10'. An artificial construct in which MTM was fused to full-length DOT1L (MTM-DOT1L) behaved similarly. These results suggest that DOT1L recruitment delays the gene silencing induced by differentiation via its transcriptional maintenance activity, but cannot establish a fully immortalized state.

$M L L-A F 10$ activates transcription by providing ENL to AF4. Since MTM-AF10' did not immortalize myeloid progenitors, but MLL-AF10' did, we inferred that the $5^{\prime}$ portion of MLL contained additional structures necessary for full transformation by MLLAF10. To identify such structures, we generated a series of constructs in which various portions of MLL were added back to MTMAF10' and examined their transforming ability (Figure 5A and Supplemental Figure 5A). A mutant ( $\left.\mathrm{P}^{\prime} 1008 / 1267-\mathrm{AF} 10^{\prime}\right)$, which included the evolutionarily conserved TRX2 domain (41) (Supplemental Figure $5 \mathrm{~B}$ ), was able to immortalize myeloid progenitors
(Figure 5A). Since MTM-ENL' fully transformed myeloid progenitors, we inferred that the TRX2 domain would associate with AF4. Indeed, fanChIP-WB analysis on a series of MLL deletion mutants demonstrated that MLL associated with AF4 or AF5Q31 through the TRX2 domain on chromatin (Figure 5B). Additionally, a region containing the conserved U1 small nuclear ribonucleoprotein (U1 snRNP) domain (Supplemental Figure 5B) also showed weak AF4-binding ability (Figure 5B, MLL 641/1007), which may further contribute to AF4 recruitment. An MLL-AF10 mutant (MLL d869/1144-AF10') lacking these AF4 interaction domains failed to fully immortalize myeloid progenitors (Figure 5A). These results indicate that MLL-AF10 activates its target genes through two key interactions: one with AF4 via the TRX2 domain and one with DOT1L via the OM-LZ domain.

Next, we performed loss-of-function studies for Dot1l and Enl using specific shRNAs on myeloid progenitors immortalized by MLL-ENL', MLL-AF10', and MLL-AF5-4. Hoxa9 expression in MLL-AF10'-transformed cells was substantially decreased by Dot1l knockdown, but not in MLL-ENL'- or MLL-AF5-4-transformed cells (Figure 5C). This suggests that DOT1L is directly involved in MLL-AF10-dependent transcriptional activation. However, clonogenicity in the following round was impaired in all immortalized cell lines, indicating that the continuous presence of endogenous DOT1L is required for the long-term maintenance of Hoxa9 expression, as previously reported (28-31). Enl knockdown markedly decreased Hoxa9 expression and clonogenicity in MLL-AF10'- and MLL-AF5-4-transformed cells, but not as severely in MLL-ENL'-transformed cells (Figure 5D), suggesting that MLL-AF10' and MLL-AF5-4 induce AEP-SL1 complex formation on chromatin through endogenous ENL protein. Consistent with these notions, an MTM-DOT1L-derivative (P'1008/1267DOT1L) construct in which the TRX2 domain was added back to MTM-DOT1L, showed full transformation ability (Supplemental Figure 5, A and C-E), while its mutant proteins lacking the major ENL-binding motifs ( $\mathrm{P}^{\prime} 1008 / 1267-D O T 1 L$ d673/1087) or an intact HMT domain (P'1008/1267-DOT1L mut) did not show this ability. These data demonstrate the importance of both the ENL-providing ability and the HMT activity of DOT1L in MLLAF10-dependent transcriptional activation.

The activities of AF4 and DOT1L cooperate in leukemogenesis. To investigate whether the activities of AEP and the DOT1L complex functionally collaborate during leukemic transformation, we performed a myeloid progenitor transformation assay by simultaneously transducing 2 genes (Figure 6A). The clonogenicity of MTM-AF5-4-transduced cells was substantially increased by the coexpression of MTM-DOT1L. Thus, AEP-dependent transcriptional activation and DOT1L-dependent transcriptional maintenance collaborate to increase clonogenicity.

Next, we examined the leukemogenic potential of various MLL-mutant constructs in vivo (Figure 6B). Neither MTM-AF5-4 nor MTM-AF10' induced leukemia in vivo within 150 days, while MTM-ENL' and MLL-AF10' induced leukemia at near-full penetrance. These results indicate that both AEP-dependent transcriptional activation and DOT1L-dependent transcriptional maintenance are required for the onset of leukemia in vivo. Thus, we conclude that the functions of both AF4 and DOT1L are required for MLL fusion-dependent leukemogenesis. 
A

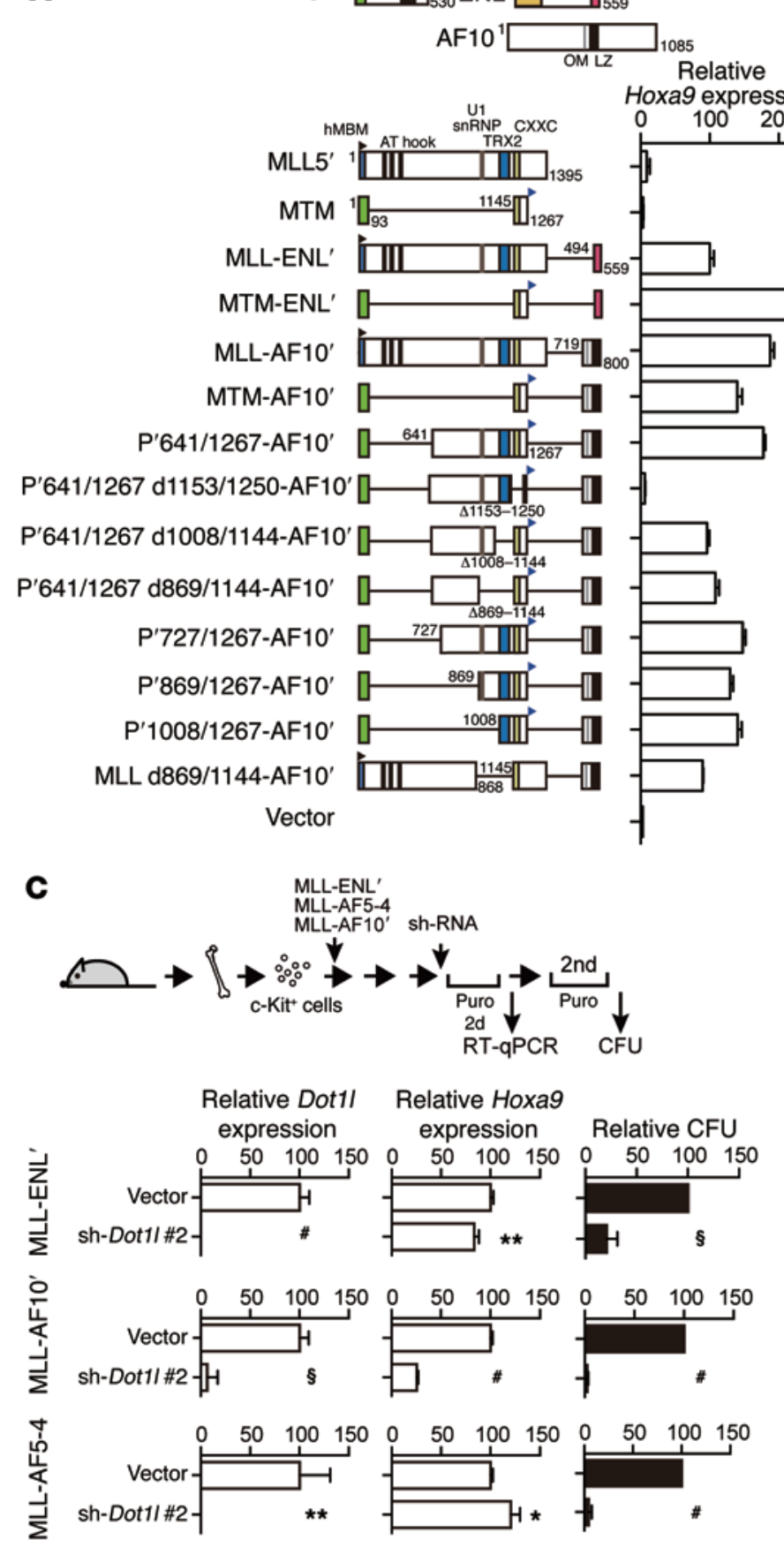

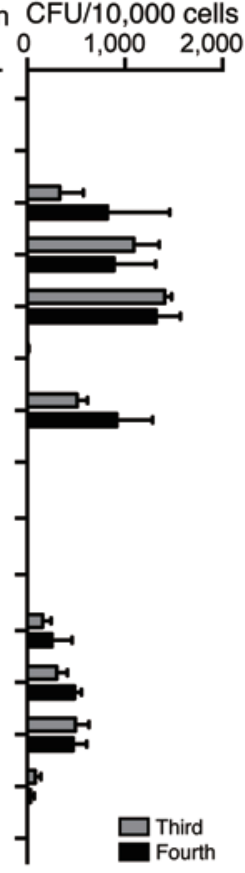

B

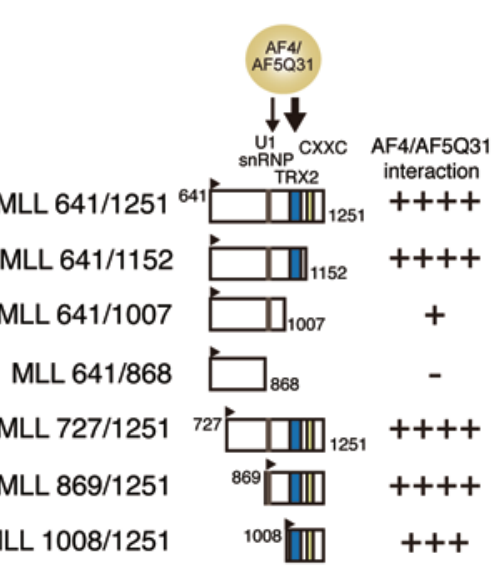

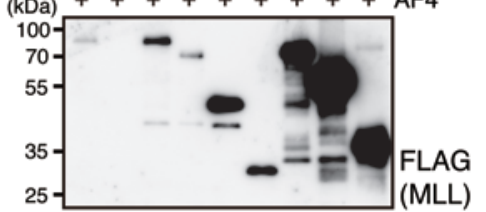

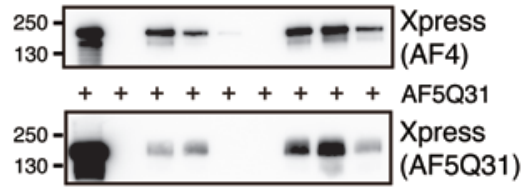

D

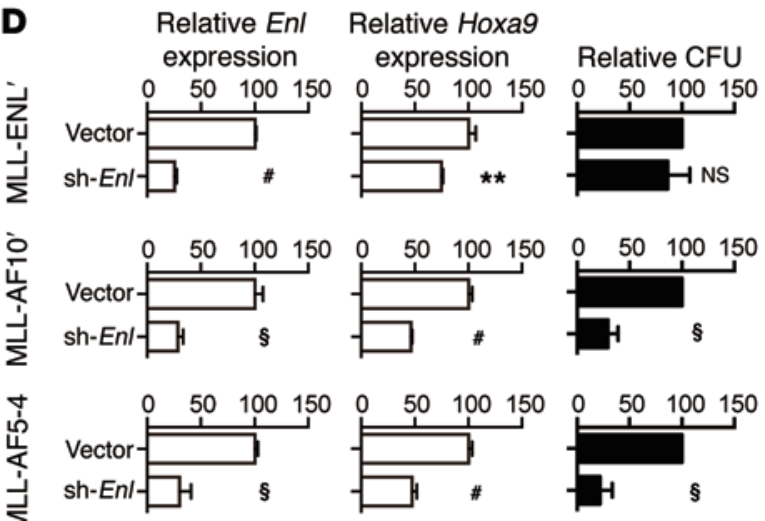

Figure 5. Mechanism of MLL-AF10-dependent transformation. (A) Structural requirement of MLL-AF10 for leukemic transformation. Various MLL-AF10 constructs were analyzed by the myeloid progenitor transformation assay, as in Figure 4D. P', PWWP. (B) Association between MLL and AF4 on chromatin 293T cells transiently expressing various FLAG-tagged MLL mutants and Xpress-tagged AF4 or AF5Q31 were analyzed by fanChIP-WB using an anti-FLAG antibody. (C) Effect of Dot1l knockdown on various MLL fusion-transformed cells. Transduced cells were selected for puromycin resistance for 2 days. RNA was extracted 3 days after transduction of shRNAs. The expression levels of Dot1/ and Hoxa9 normalized to Gapdh (representative of 2 independent experiments) are shown as the value relative to that of the vector control (set at 100\%). Error bars represent the SD of PCRs performed in triplicate. CFU relative to those of the vector control in the same experiment (set at $100 \%$ ) is shown with error bars (SD for $>3$ independent experiments). ${ }^{*} P \leq 0.05$, ${ }^{*} P \leq 0.01$, $\S P \leq 0.001$, and ${ }^{\#} P \leq 0.0001$, by unpaired, 2 -tailed $t$ test, comparing each sample with the vector control. (D) Effect of Enl knockdown on various MLL fusion-transformed cells. Experiments were performed using shRNA for Enl as shown in panel $\mathbf{C}$. ${ }^{* *} P \leq 0.01,{ }^{\circledR} P \leq 0.001$, and ${ }^{\#} P \leq 0.0001$, by by unpaired, 2-tailed $t$ test, comparing each sample with the vector control. 

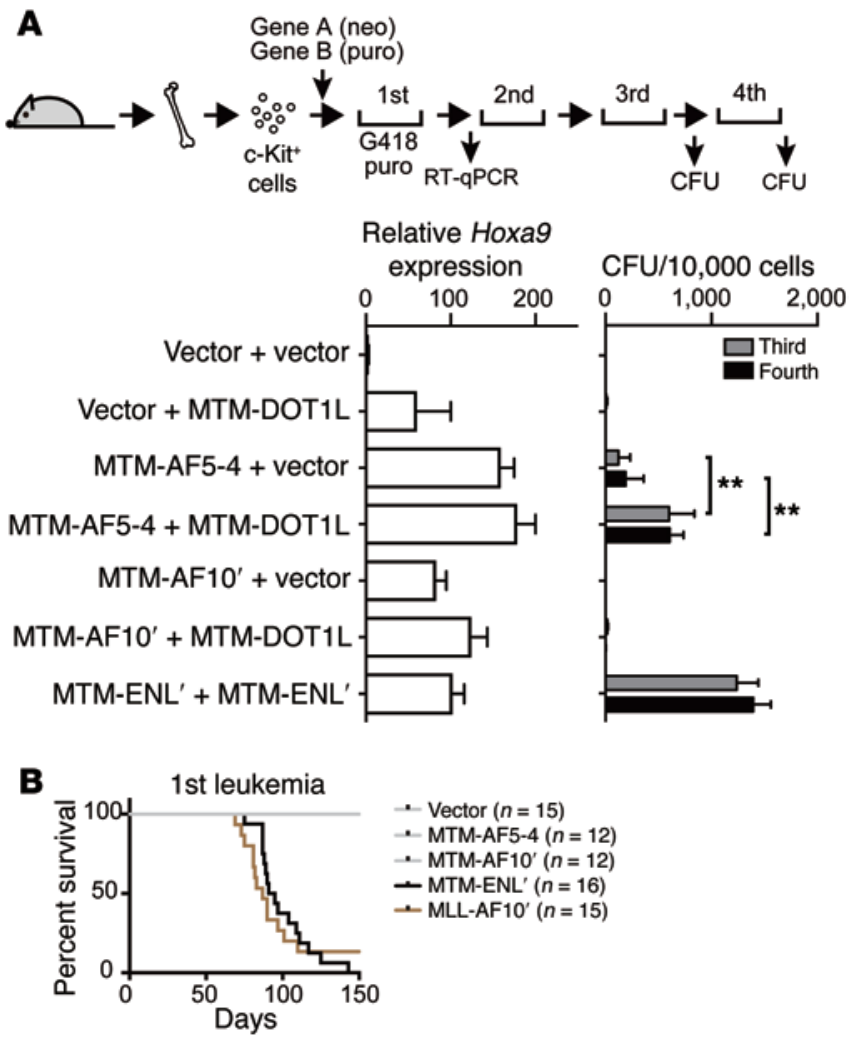

Figure 6. Recruitment of both AF4 and DOT1L activities is required for MLL fusion-dependent leukemogenesis. (A) Cooperative transformation by recruitment of AF4 and DOT1L activities. Doubly transduced cells were selected in the first round. Hoxa9 expression and colony-forming activity were analyzed as in Figure 4D. ${ }^{* *} P \leq 0.01$, by ordinary 1-way ANOVA, comparing the indicated sample pairs. neo, neomycin. (B) Leukemogenic potential of various constructs in vivo. Survival of mice transplanted with hematopoietic progenitors transduced with the indicated transgenes. $n$ values indicate the number of mice analyzed.

Simultaneous inhibition of MENIN and DOT1L efficiently eradicates MLL cells. Because AF4 recruitment appears to be primarily dependent on MLL-ENL (Figure 3) and target recognition by MLL fusion proteins requires $\operatorname{MENIN}(4,5)$, we hypothesized that MI-2-2, a MENIN-MLL interaction inhibitor (42), would inhibit AF4 recruitment to the MLL target chromatin in MLL-AEP leukemia cells. Indeed, MI-2-2 impaired the expression of HOXA9 and MEIS1 in NODAL modulator 1 (NOMO-1) cells, which express MLL-AF9 endogenously (Figure 7, A and B). There was no effect on the expression of either gene by a near-complete knockdown of WT MLL, indicating that the effects of MI-2-2 could be mostly attributed to the disruption of the MLL-AF9-MENIN complex. Given the collaborative effects of AEP and DOT1L on MLL fusion-dependent gene activation, we hypothesized that the combinatorial use of a MENIN inhibitor and a DOT1L inhibitor might have synergistic effects on leukemic cell growth. MI-2-2 and EPZ5676, a DOT1L HMT inhibitor (34), each reduced clonogenicity by approximately $30 \%$ to $40 \%$ at $3 \mu \mathrm{M}$ in leukemia cells induced by MLL-ENL in vivo (Figure 7C). The colony-forming ability of these leukemia cells ex vivo represents the frequency of selfrenewing leukemia-initiating cells $(3,43)$. Concomitant exposure to both compounds at this concentration resulted in efficient erad- ication of the clonogenic cells. Similar results were obtained with MLL-AF9-, MLL-AF10-, and MLL-AF5-4-transformed cells, but not with E2A-HLF-transformed cells (Figure 7D). MV4-11 cells, which express MLL-AF4 endogenously, were also susceptible to the double exposure (Supplemental Figure 6, A and B). Exposure of MLL-ENL cells to these 2 compounds at these suboptimal concentrations for only 3 days effectively decreased MLL target gene expression and induced the expression of $C d 11 b$, which is a differentiation marker (Figure 7E). A transplantation experiment following the 3-day exposure ex vivo showed that the combined drug treatment effectively attenuated the leukemogenic potential in vivo (Figure 7F). Thus, therapeutics against these molecular targets that simultaneously inhibit DOT1L and MENIN may provide better treatment for patients than do single drugs.

\section{Discussion}

We showed that MLL fusion proteins, such as MLL-ENL and MLLAF10, exert 2 separate actions to cause leukemia in vivo: AEPdependent transcriptional activation and DOT1L-dependent transcriptional maintenance. Although PRC1 can be recruited to some MLL-ENL target loci, MLL fusion proteins appear to function primarily in the absence of PRC1.

ENL binds specifically to chromatin containing H3K9/18/27ac via the YEATS domain (17). Localization of ENL is also closely correlated with the presence of the DOT1L-AF10 family complex (Figure 1, E and F), which associates with chromatin containing unmodified H3K27 (39). Given observations, we propose the following model (Figure 8A): (a) ENL forms a metastable complex with the DOT1L-AF10 family complex on chromatin containing unmodified H3K27; (b) upon acetylation of nearby nucleosomes, the ENL-containing DOT1L complex binds to H3K9/18/27ac marks via the YEATS domain; (c) this complex presents ENL to $\mathrm{AF} 4$ proteins to form AEP on the chromatin; and (d) the chromatin-bound AEP further recruits SL1 to initiate transcription, while the DOT1L-AF10 family complex inhibits SIRT1-dependent transcriptional repression by methylating lysine 79 on histone $\mathrm{H} 3$ of the nearby chromatin. Since AHD binds to AF4 proteins with a higher affinity than it does to DOT1L and has an intrinsically disordered structure that facilitates the exchange of interacting partners (36), MLL-ENL primarily associates with AEP components on chromatin (Figure 8B).

The mechanism by which MLL-AF10 activates gene expression and transforms hematopoietic progenitors was previously unclear and controversial $(11,26)$. The results from this study show that simple recruitment of DOT1L to target promoters only prolongs the expression of target genes for a limited period (Figure 4D). We discovered that the TRX2 domain of MLL, which recruits $\mathrm{AF} 4$, is additionally required for MLL-AF10-dependent transformation (Figure 5, A and B). The results of a structural and functional analysis of artificial MLL-DOT1L fusion proteins indicated that DOT1L exerts its transforming potential through its ENL-binding motifs (Supplemental Figure 5, C-E). These results suggest that the MLL-AF10-DOT1L complex functions both as an ENL provider for AF4 to efficiently load AEP onto chromatin and as a chromatin modifier. Thus, we propose a model in which MLLAF10 recruits ENL through DOT1L and then provides it to AF4 to induce AEP-SL1 complex formation (Figure 8C). 
A
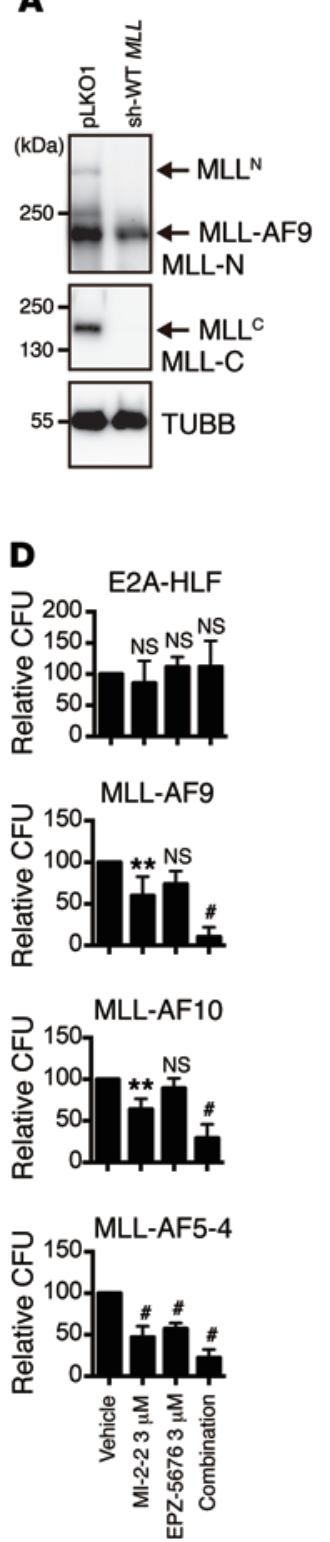
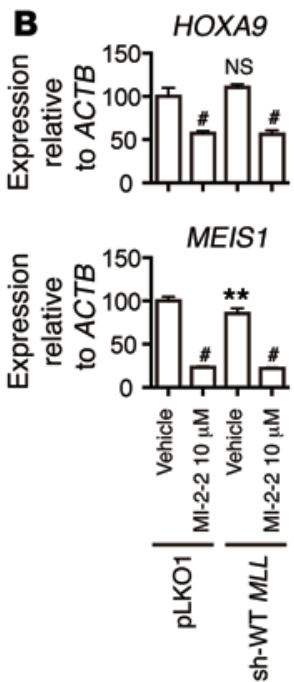

E
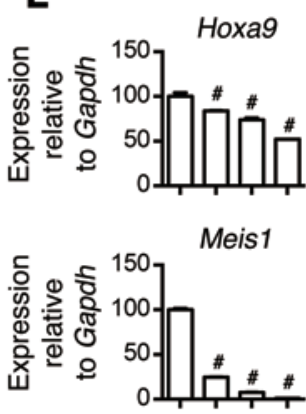

Cd11b

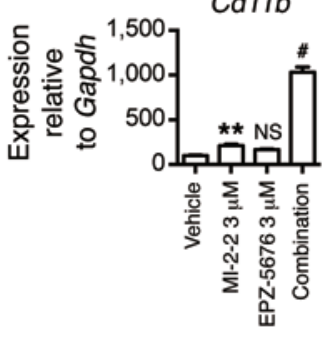

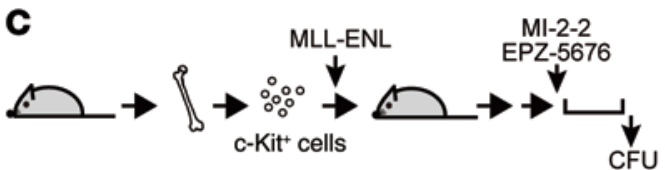

MI-2-2

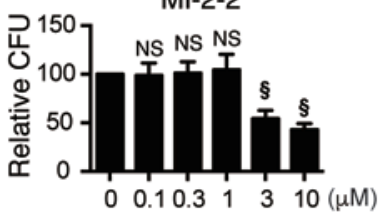

EPZ-5676
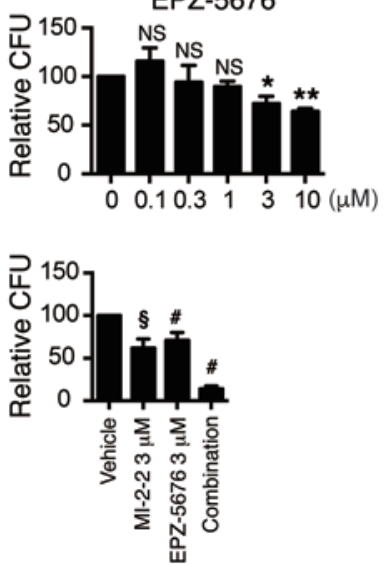

EPZ-5676 $(3 \mu \mathrm{M})$

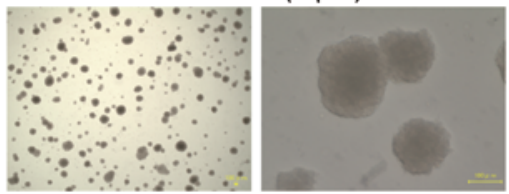

MI-2-2 (3 $\mu \mathrm{M})$ EPZ-5676 (3 $\mu \mathrm{M})$

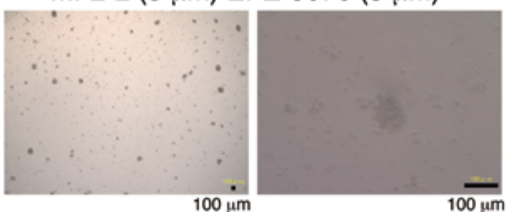

$\mathbf{F}$
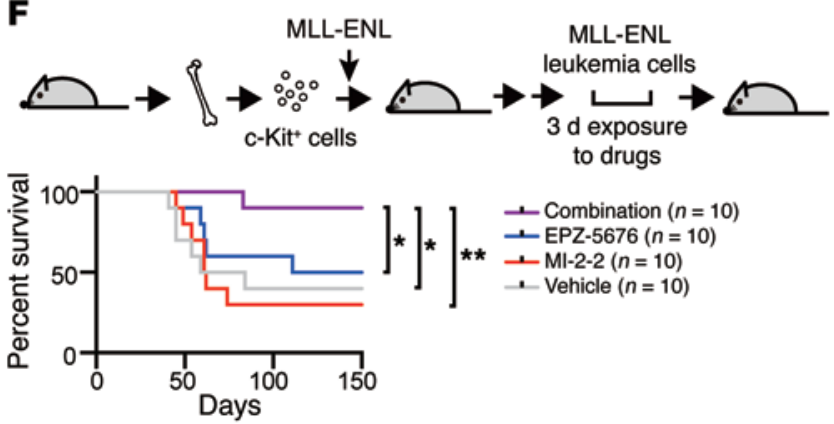

Figure 7. Combined antileukemic effects of the MENIN-MLL interaction inhibitor and the DOT1L HMT inhibitor. (A) Knockdown of WT MLL in NOMO-1

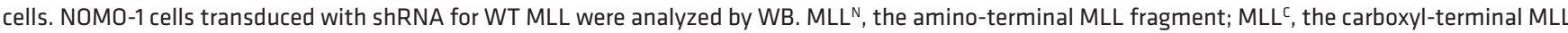
fragment. (B) Effect of MI-2-2 in NOMO-1 cells. NOMO-1 cells in A were cultured in the presence of MI-2-2 (10 $\mu \mathrm{M})$ for 1 day and analyzed by RT-qPCR. The expression level, normalized to ACTB (representative of 2 independent experiments), is shown as the value relative to that of the vector/vehicle control (set at $100 \%$ ). Error bars represent the SD of PCRs performed in triplicate. ${ }^{*} P \leq 0.01$ and ${ }^{*} P \leq 0.0001$, by ordinary 1 -way ANOVA, comparing each sample with the vector/vehicle control. (C) Combined effects of MI-2-2 and EPZ-5676 on MLL-ENL leukemia cells. Murine MLL-ENL leukemia cells were cultured in the presence of the drugs at the indicated concentrations ex vivo. CFU relative to those of the vehicle control (set at $100 \%$ ) are shown with error bars (SD of $>3$ independent experiments). ${ }^{*} P \leq 0.05,{ }^{* *} P \leq 0.01,{ }^{\$} P \leq 0.001$, and ${ }^{\#} P \leq 0.0001$, by ordinary 1-way ANOVA, comparing each sample with the vehicle control. Images of colonies on day 6 are shown. Scale bars: $100 \mu \mathrm{m}$. (D) Combined effects of MI-2-2 and EPZ-5676 on various immortalized progenitors. Murine myeloid progenitors immortalized by various transgenes were analyzed, as in $\mathbf{C}$. ${ }^{*} P \leq 0.01$ and ${ }^{\#} P \leq 0.0001$, by ordinary 1 -way ANOVA, comparing each sample with the vehicle control. (E) Gene expression after exposure to MI-2-2 and/or EPZ-5676. RT-qPCR analysis was performed on MLL-ENL leukemia cells after 3 days of drug treatment. The expression level normalized to Gapdh (representative of 2 independent experiments) is shown as the value relative to that of the vehicle control (set at $100 \%$ ). Error bars represent the SD of PCRs performed in triplicate. ${ }^{* *} P \leq 0.01$ and ${ }^{\#} P \leq 0.0001$, by ordinary 1-way ANOVA, comparing each sample with the vehicle control. (F) Combined effects of MI-2-2 and EPZ-5676 on the leukemia stem cell potential. MLLENL leukemia cells treated with the drugs for 3 days were transplanted into sublethally irradiated syngeneic mice. ${ }^{*} P \leq 0.05$ and ${ }^{* *} P \leq 0.01$, by log-rank test for the indicated pairs. 

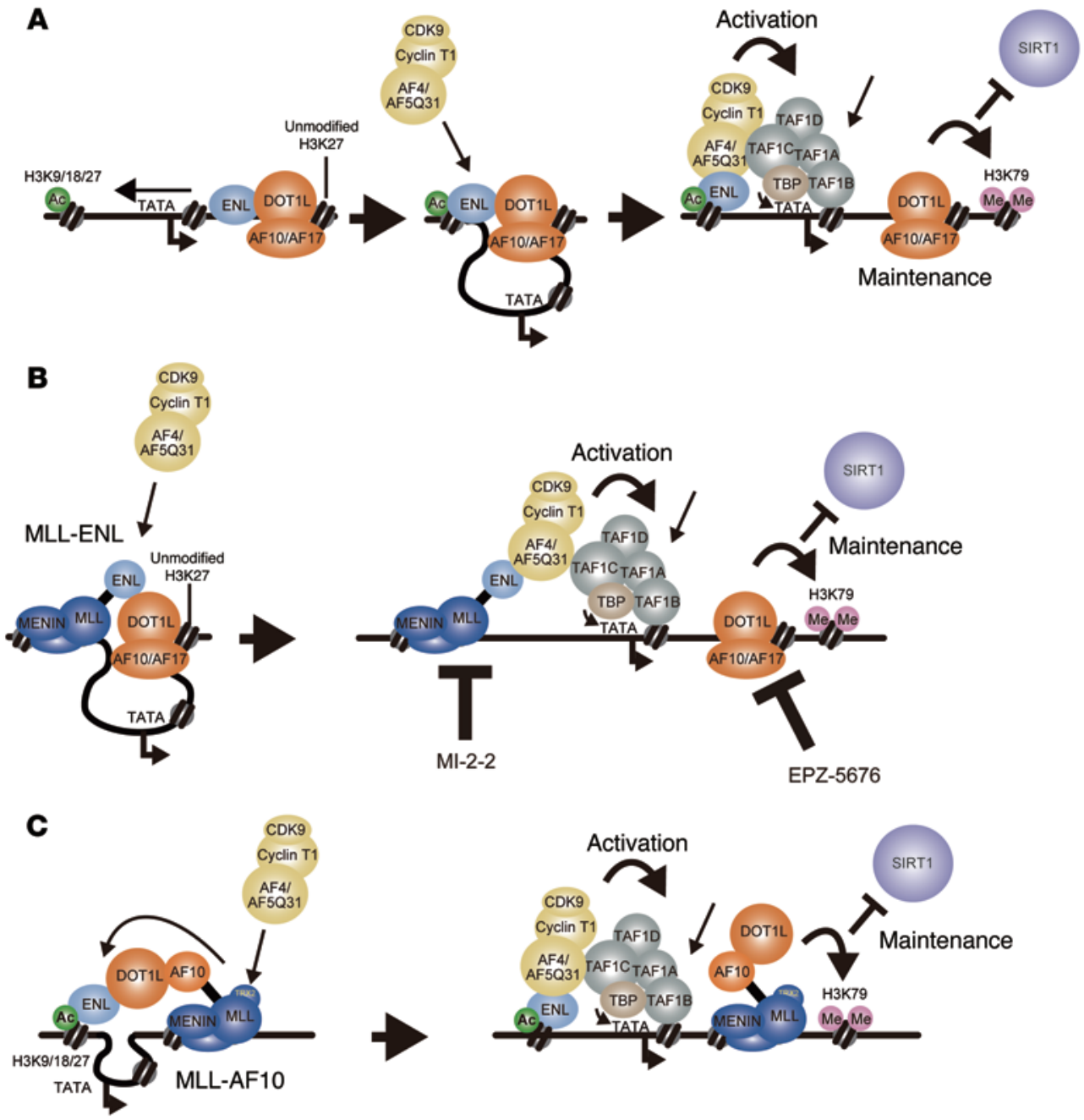

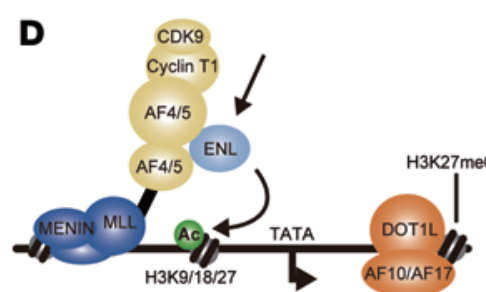

MLL-AF4/5

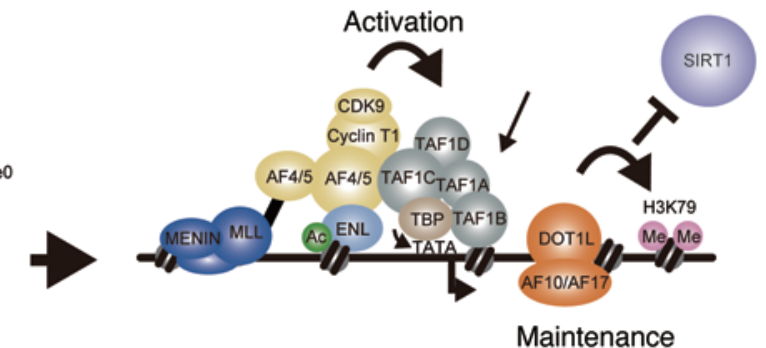

Figure 8. Working models of gene activation. Working models of gene activation by AEP (A), MLL-ENL (B), MLLAF10 (C), and MLL-AF4/5Q31 (D). Me, methylated.

The artificial construct in which MTM was fused with the AF4-binding platform (MTM-AF5-4) activated MLL target genes and transformed myeloid progenitors ex vivo (Figure 4D), but failed to cause leukemia in vivo (Figure 6B). In contrast, MTM-ENL', which could facilitate both AF4-dependent transcriptional activation and DOT1L-dependent transcriptional maintenance, efficiently induced leukemia, indicating that both activation and maintenance of the MLL target genes are required for the onset of leukemia in vivo. These 2 activities likely feed off each other, as the DOT1L-dependent transcriptional maintenance activity protects H3K9/18/27ac from SIRT1 (24) to set the stage for subsequent AEP-SL1 complex formation (Figure 8A), while transcription-coupled ubiquitination of histone

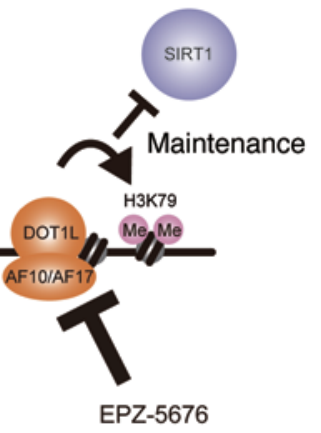

H2B lysine 120 (H2BK120) stimulates DOT1L-dependent H3K79 methylation (44). Consistent with this hypothesis, RNF20, an H2BK12O ubiquitin ligase, has been shown to play important roles in the continuous growth of MLL-AF9 leukemia cells (45). MLL-AF4 leukemia cells contain MLL target chromatin enriched with H3K79me2 and depend on DOT1L for their proliferation (46), indicating that the endogenous DOT1L complex plays a significant role in leukemic transformation by MLL-AF4 (Figure 8D).

The requirement of AF4-binding platforms for MLL fusiondependent transformation (20) indicates that the AF4 activity is recruited by the MLL fusionMENIN complex, while the DOT1L activity appears to be recruited to the MLL target loci either directly or indirectly $(11,25,28-30,32$, $46,47)$. These 2 activities cooperatively increase clonogenicity in MLL cells (Figure 6). Simultaneous exposure to the MENIN-MLL interaction inhibitor (42) and the DOT1L HMT inhibitor (34), which would induce dissociation of the MLL fusion-MENIN-AF4 complex from target chromatin and loss of the DOT1L HMT activity, eradicated the leukemia-initiating cells more efficiently than did the use of a single drug (Figure 7F), providing a rationale for the simultaneous administration of these 2 drugs to improve treatment efficacy. Our results suggest that WT MLL may mediate AEP recruitment via its TRX2 domain in non- $M L L$-r conditions. NPM1 mutation-associated leukemia, in which WT MLL activates HOXA9 and MEIS1, is also susceptible to the combinatorial use of the MENIN-MLL interaction inhibitor and the DOT1L HMT inhibitor (48), suggesting that the MLL/AEP axis and DOT1L activity also cooperate in nucleophosmin (NPM1) mutation-associated leukemia. Because of the cooperative nature of transcriptional activation and maintenance, the combinatorial use of 2 reagents that inhibit these 2 axes may improve the clinical outcome compared with the use of a single reagent.

\section{Methods}

Vector construction. The pMSCV-neo-MLL-ENL, MLL-AF10, and E2AHLF vectors and their derivatives have been previously described (6). 
Various new gene constructs were generated by restriction enzyme digestion/PCR-based mutagenesis. The cDNAs were cloned into the pMSCV neo vector (for virus production) (Clontech) or into the pCMV5 vector and the pcDNA4 HisMax vector (for transient expression). The pLKO-zeo-TK-RL reporter and the pLKO-puro-FR-LUC (or blaFR-LUC) reporter vectors have been previously described (20). The shRNA expressionvectors, targeting murine Enl(TRCN0000084405); Taf1c (TRCNO000082215); Dot1l no. 1 (TRCN00000125101); Dot1l no. 2 (TRCNO000125099); human DOT1L (TRCNO000020212); MLL (TRCNO000234743); ENL no. 1 (TRCNO000019291; ENL no. 2 (TRCNO000019293); and TAF1D (TRCNO000134377), were obtained from GE Healthcare.

Cells and cell culture. The human leukemia cell lines K562 (JCRB Cell Bank), HB1119 (40, 49), and NOMO-1 (JCRB Cell Bank) were cultured in RPMI 1640 medium supplemented with 10\% FBS and penicillin-streptomycin (PS). The MV4-11 (ATCC) cell line was cultured in Iscove's Modified Dulbecco's Medium (IMDM) supplemented with $10 \%$ FBS and PS. The 293T and 293TN (System Biosciences) cell lines and iMEFs (20) were cultured in DMEM supplemented with $10 \%$ FBS and PS. Ecotropic virus-packaging cells (PLAT-E cells, a gift of Toshio Kitamura, The University of Tokyo, Tokyo, Japan) (50) were cultured in DMEM supplemented with 10\% FBS, puromycin, blasticidin, and PS. 293TL cells, which carry the GAL4-responsive luciferase reporter, were generated by transduction of the pLKO-puro-FR-LUC reporter (20). 293TL cells stably expressing various FLAG-tagged GAL4 (fGAL4) fusion proteins were generated by sequential transduction of the pLKO-zeo-TK-RL reporter, the pLKO-bla-FR-LUC reporter, and the pMSCV-hygro-fGAL4 fusion vectors.

nucfrIP. Subcellular fractions of 293T cells were obtained by cytoskeleton (CSK) buffer extraction and micrococcal nuclease (MNase) treatment as previously described (6). $293 \mathrm{~T}$ cells cultured in a $10-\mathrm{cm}$ dish were resuspended in $1 \mathrm{ml} \mathrm{CSK}$ buffer $(100 \mathrm{mM} \mathrm{NaCl}, 10 \mathrm{mM}$ PIPES, pH 6.8, 3 mM MgCl 2 , 1 mM EGTA, pH 7.6, 0.3 M sucrose, 0.5\% Triton X-100, $5 \mathrm{mM}$ sodium butyrate, $0.5 \mathrm{mM}$ DTT, and EDTA-free protease inhibitor cocktail [Roche]), incubated on ice for 5 minutes, and then centrifuged $\left(400 \times g, 4^{\circ} \mathrm{C}, 4 \mathrm{~min}\right)$. The supernatant was removed, and the pellet was resuspended in $1 \mathrm{~m}$ MNase buffer $(50 \mathrm{mM}$ Tris-HCl, pH 7.5, $4 \mathrm{mM} \mathrm{MgCl}_{2}, 1 \mathrm{mM} \mathrm{CaCl}_{2}, 0.3 \mathrm{M}$ sucrose, $5 \mathrm{mM}$ sodium butyrate, $0.5 \mathrm{mM}$ DTT, and protease inhibitor cocktail). One unit of MNase (Sigma-Aldrich) was added to the suspension, and the mixture was incubated at $37^{\circ} \mathrm{C}$ for 10 to 12 minutes to obtain mononucleosomes. The MNase reaction was stopped by adding EDTA (pH 8.0) at a final concentration of $20 \mathrm{mM}$. The reaction mixture was centrifuged $\left(17,000 \times g, 4^{\circ} \mathrm{C}, 5 \mathrm{~min}\right.$ ) to separate the supernatant (nucleosome fraction) and the pellet. The nucleosome fractions were subjected to IP using specific antibodies (Supplemental Table 1), washed 5 times with MNase buffer containing $20 \mathrm{mM}$ EDTA, and then eluted in elution buffer ( $1 \%$ SDS and $50 \mathrm{mM} \mathrm{NaHCO}$ ). The eluted material was analyzed by Western blotting and MS.

fanChIP. The chromatin fractions of 293T derivatives and HB1119 cells were prepared as previously described (6). Cells were suspended in CSK buffer and centrifuged to remove the soluble fraction in the same manner as for the nucfrIP analysis. The pellet was resuspended in MNase buffer and treated with MNase at $37^{\circ} \mathrm{C}$ for 3 to 6 minutes to obtain oligonucleosomes. The MNase reaction was stopped by adding EDTA ( $\mathrm{pH} \mathrm{8.0)}$ at a final concentration of $20 \mathrm{mM}$. Lysis buffer $(250 \mathrm{mM} \mathrm{NaCl}, 20 \mathrm{mM}$ sodium phosphate, $\mathrm{pH}$ 7.0, $30 \mathrm{mM}$ sodium pyrophosphate, $5 \mathrm{mM}$ EDTA, $10 \mathrm{mM} \mathrm{NaF}$, 0.1\% NP-40, 10\% glycerol, 1 mM DTT, and EDTA-free protease inhibitor cocktail) was then added to increase the solubility. The chromatin fraction was cleared by centrifugation and subjected to IP with specific antibodies (Supplemental Table 1) and magnetic microbeads (Protein G Magnetic Beads; Invitrogen, Thermo Fisher Scientific) or with anti-FLAG M2 antibody-conjugated beads. The precipitates were washed 5 times with washing buffer (1:1 mixture of lysis buffer and MNase buffer with $20 \mathrm{mM}$ EDTA) and then eluted in elution buffer. The eluted material was analyzed by various methods including Western blotting, qPCR, and deep sequencing. The fanChIP-WB analysis was performed using 293T-derivative cell lines.

Western blotting. Western blotting was performed as previously described (51). The antibodies used in this study are listed in Supplemental Table 1.

Fractionation-assisted cross-linked ChIP. For fractionation-assisted cross-linked ChIP (faxChIP), cells were cross-linked by a 5-minute incubation with $5 \%$ formaldehyde. Glycine was added to stop the cross-linking reaction at a final concentration of $125 \mathrm{mM}$. After rinsing with PBS, cells were suspended in CSK buffer and centrifuged to remove the soluble fraction in the same manner as for the fanChIP protocol. The pellet was resuspended in MNase buffer and treated with MNase at $37^{\circ} \mathrm{C}$ for 3 to 6 minutes to obtain oligonucleosomes. The MNase reaction was stopped by adding EDTA (pH 8.0) at a final concentration of $20 \mathrm{mM}$. The chromatin was solubilized by adding SDS at a final concentration of $1 \%$ and incubated on ice for 10 minutes. The chromatin fraction was cleared by centrifugation and mixed with 2 volumes of IP dilution buffer (0.01\% SDS, 1.1\% Triton-X-100, 1.2 mM EDTA, pH 8.0, 16.7 mM Tris$\mathrm{HCl}, \mathrm{pH} 7.6,167 \mathrm{mM} \mathrm{NaCl}$, and EDTA-free protease inhibitor cocktail) and subjected to IP, as in the fanChIP protocol. faxChIP was applied for the ChIP analysis of H3K79me2 and H3K27me3.

ChIP-qPCR and ChIP-seq. The eluted material obtained by fanChIP (or faxChIP) was extracted by phenol/chloroform/isoamyl alcohol. DNA was precipitated with glycogen (Nacalai Tesque) and analyzed by qPCR and deep sequencing. Deep sequencing of the precipitated DNA was performed using a TruSeq ChIP Sample Prep Kit (Illumina) and a Genome Analyzer IIx (Illumina) or a HiSeq 2000 (Illumina) at the core facility of Hiroshima University (Hiroshima, Japan). The data were visualized using the Integrative Genome Viewer (The Broad Institute). qPCR analysis of the precipitated DNA was performed using the custom-made primer sets listed in Supplemental Table 2. The value relative to the input was determined using a standard curve and the relative quantification method.

Virus production. The ecotropic retrovirus was produced using PLAT-E packaging cells (50). The lentivirus was produced in 293TN cells using the pMDLg-pRRE, pRSV-rev, and pMD2.G vectors (52). The supernatant medium containing the virus was harvested $24-48$ hours following transfection and used in viral transduction.

Myeloid progenitor transformation assay. The myeloid progenitor transformation assay was performed as previously described (6). Bone marrow cells were harvested from the femurs and tibiae of 5-weekold female C57BL/6 mice (purchased from CLEA Japan Inc.). c-Kitpositive cells were enriched using magnetic beads conjugated with an anti-c-Kit antibody (Miltenyi Biotec), transduced with a recombinant retrovirus by spinoculation, and then plated on a methylcellulose medium (IMDM containing 20\% FBS, 1.6\% methylcellulose, and 100 $\mu \mathrm{M} \beta$-mercaptoethanol) containing murine stem cell factors, IL-3, and 
granulocyte-macrophage CSF (10 ng/ml of each), G418 (1 mg/ml), and/or puromycin $(2 \mu \mathrm{g} / \mathrm{ml})$, which were added to the first round of culture to select for transduced cells. Hoxa 9 was quantified by reverse transcription-qPCR after the first round of culture. CFU were quantified per $10^{4}$ plated cells after 4 to 6 days in culture.

In vivo leukemogenesis assay. c-Kit-positive cells $\left(2 \times 10^{5}\right)$ prepared from mouse femurs and tibiae were transduced with retrovirus by spinoculation and intravenously transplanted into sublethally irradiated (6 Gy) C57BL/6 mice. Moribund mice were euthanized, and the isolated cells were cultured for more than 3 passages in the methylcellulose medium used for the myeloid progenitor transformation assay to remove untransformed cells and then subjected to secondary transplantation. Leukemia cells $\left(2 \times 10^{5}\right)$ cultured ex vivo, with or without the exposure to drugs, were transplanted in the same manner as for the primary transplantation.

Transactivation assay. The expression vectors for the various GAL4 fusion proteins were transfected in 293TL cells along with pRLtk plasmid (Promega). Luciferase/Renilla luciferase activity was measured using the Dual-Luciferase Reporter Assay System (Promega). Luciferase activity values were normalized to Renilla luciferase activity and expressed as the mean and SD of triplicate samples.

RNA-seq. Total RNA was prepared using the RNeasy Kit (QIAGEN) and the quality assessed using a eukaryote Bioanalyzer RNA Nano Kit (Agilent Technologies). Deep sequencing of total RNA was performed using a SureSelect Strand Specific RNA Library Prep Kit (Agilent Technologies) and a Genome Analyzer IIx (Illumina) at the core facility of Hiroshima University. Gene expression was normalized as reads per kilobase of exon per million mapped (RPKM), with the cutoff value set to 5 in the vector control. Gene set enrichment analysis (GSEA) was carried out using the preranked method, with 1,000 permutations with the gene sets.

Liquid chromatography-tandem MS analysis. Trypsin digestion of proteins was performed as previously described (6). Tandem MS (MS/ MS) analysis was performed using an LTQ Orbitrap ELITE ETD mass spectrometer (Thermo Fisher Scientific). The methods used for liquid chromatography-MS/MS (LC-MS/MS) were slightly modified from those described previously (53). The mass spectrometer was operated in data-dependent acquisition mode, in which MS acquisition with a mass range of 400 to $1,000 \mathrm{~m} / z$ was automatically switched to MS/ MS acquisition under the control of Xcalibur software (Thermo Fisher Scientific). The top 4 precursor ions in the MS scan were selected by Orbitrap, with a resolution of $R=240,000$, and the ions in subsequent $\mathrm{MS} / \mathrm{MS}$ scans were analyzed with an ion trap in automated gain control (AGC) mode, in which AGC values were $1 \times 10^{6}$ and $1.00 \times 10^{4}$ for full MS and MS/MS, respectively. For fragmentation, electron transfer dissociation (ETD) was used.

$R T-q P C R$. RNA was prepared using the RNeasy Kit (QIAGEN) and reverse transcribed using a Superscript III First Strand cDNA Synthesis Kit with oligo(dT) primers (Life Technologies, Thermo Fisher Scientific). Gene expression was confirmed by qPCR using the TaqMan probes described in Supplemental Table 3 (Life Technologies, Thermo Fisher Scientific). The expression levels, normalized to those of Gapdh, Actb, or Tbp for mouse transcripts and ACTB or TBP for human transcripts, were determined using a standard curve and the relative quantification method as described in ABI User Bulletin no. 2.

Cell viability assay. Five thousand MV4-11 cells were cultured in 96-well plates with MI2-2 and/or EPZ-5676 in IMDM containing 10\% FBS and PS for 5 days. After incubation, cell survival was measured with the PrestoBlue Cell Viability Reagent (Thermo Fisher Scientific). The drugs were dissolved in DMSO to a final DMSO concentration of $0.2 \%$.

Accession numbers. The deep-sequencing data from the RNA-seq and ChIP-seq analyses have been deposited in the DNA Data Bank of Japan (DDBJ) Sequence Read Archive under accession numbers DRA004871 through DRA004875.

Statistics. Statistical significance was calculated by unpaired, 2-tailed $t$ test (for comparisons between 2 samples); ordinary 1-way ANOVA (for ungrouped multiple comparisons); or log-rank test (for survival curves) using GraphPad Prism 6.0 (GraphPad Software). $P$ values of greater than 0.05 were considered nonsignificant.

Study approval. All experiments involving animals were approved by the IACUC of Kyoto University.

\section{Author contributions}

AY conceived the project. HO, BS, ST, and AY performed most of the experiments. ATK provided essential reagents. TK performed the mass spectrometric analysis. HM performed deep sequencing, and AK analyzed the deep-sequencing data. AY wrote the manuscript.

\section{Acknowledgments}

This study was supported by a Japan Society for the Promotion of Science (JSPS) KAKENHI grant (16H05337, to AY). This work was also supported in part by research funding from Dainippon Sumitomo Pharma Co., Ltd.

Address correspondence to: Akihiko Yokoyama, Tsuruoka Metabolomics Laboratory, National Cancer Center, Mizukami 246-2, Kakuganji, Tsuruoka, Yamagata 997-0052, Japan. Phone: 81.235. 64.0980; E-mail: ayokoyam@ncc-tmc.jp.
1. Li BE, Ernst P. Two decades of leukemia oncoprotein epistasis: the MLL1 paradigm for epigenetic deregulation in leukemia. Exp Hematol. 2014;42(12):995-1012.

2. Krivtsov AV, et al. Transformation from committed progenitor to leukaemia stem cell initiated by MLL-AF9. Nature. 2006;442(7104):818-822.

3. Somervaille TC, Cleary ML. Identification and characterization of leukemia stem cells in murine MLL-AF9 acute myeloid leukemia. Cancer Cell. 2006;10(4):257-268.

4. Yokoyama A, Somervaille TC, Smith KS, Rozenblatt-Rosen O, Meyerson M, Cleary ML. The menin tumor suppressor protein is an essential oncogenic cofactor for MLL-associated leukemogenesis. Cell. 2005;123(2):207-218.

5 . Yokoyama A, Cleary ML. Menin critically links MLL proteins with LEDGF on cancer-associated target genes. Cancer Cell. 2008;14(1):36-46.

6. Okuda $\mathrm{H}$, et al. MLL fusion proteins link transcriptional coactivators to previously active CpG-rich promoters. Nucleic Acids Res. 2014;42(7):4241-4256.

7. Birke M, Schreiner S, García-Cuéllar MP, Mahr K, Titgemeyer F, Slany RK. The MT domain of the proto-oncoprotein MLL binds to CpG-containing
DNA and discriminates against methylation. Nucleic Acids Res. 2002;30(4):958-965.

8. Eidahl JO, et al. Structural basis for high-affinity binding of LEDGF PWWP to mononucleosomes. Nucleic Acids Res. 2013;41(6):3924-3936.

9. Zhu L, et al. ASH1L links histone H3 lysine 36 dimethylation to MLL leukemia. Cancer Discov. 2016;6(7):770-783.

10. Meyer C, et al. The MLL recombinome of acute leukemias in 2013. Leukemia. 2013;27(11):2165-2176.

11. Yokoyama A, Lin M, Naresh A, Kitabayashi I, Cleary ML. A higher-order complex containing AF4 and ENL family proteins with P-TEFb facili- 
tates oncogenic and physiologic MLL-dependent transcription. Cancer Cell. 2010;17(2):198-212.

12. Mueller D, et al. A role for the MLL fusion partner ENL in transcriptional elongation and chromatin modification. Blood. 2007;110(13):4445-4454.

13. Mohan M, et al. Linking H3K79 trimethylation to Wnt signaling through a novel Dot1-containing complex (DotCom). Genes Dev. 2010;24(6):574-589.

14. Lin C, et al. AFF4, a component of the ELL/ $\mathrm{P}$-TEFb elongation complex and a shared subunit of MLL chimeras, can link transcription elongation to leukemia. Mol Cell. 2010;37(3):429-437.

15. Sobhian B, et al. HIV-1 Tat assembles a multifunctional transcription elongation complex and stably associates with the 7SK snRNP. Mol Cell. 2010;38(3):439-451.

16. He N, et al. HIV-1 Tat and host AFF4 recruit two transcription elongation factors into a bifunctional complex for coordinated activation of HIV-1 transcription. Mol Cell. 2010;38(3):428-438.

17. Li Y, et al. AF9 YEATS domain links histone acetylation to DOT1L-mediated H3K79 methylation. Cell. 2014;159(3):558-571.

18. Peterlin BM, Price DH. Controlling the elongation phase of transcription with P-TEFb. Mol Cell. 2006;23(3):297-305

19. Shilatifard A, Lane WS, Jackson KW, Conaway RC, Conaway JW. An RNA polymerase II elongation factor encoded by the human ELL gene. Science. 1996;271(5257):1873-1876.

20. Okuda H, Kanai A, Ito S, Matsui H, Yokoyama A. AF4 uses the SL1 components of RNAP1 machinery to initiate MLL fusion- and AEP-dependent transcription. Nat Commun. 2015;6:8869.

21. Okuda H, Takahashi S, Takaori-Kondo A, Yokoyama A. TBP loading by AF4 through SL1 is the major rate-limiting step in MLL fusion-dependent transcription. Cell Cycle. 2016;15(20):2712-2722.

22. Feng $\mathrm{Q}$, et al. Methylation of $\mathrm{H} 3$-lysine 79 is mediated by a new family of HMTases without a SET domain. Curr Biol. 2002;12(12):1052-1058.

23. Jones $\mathrm{B}$, et al. The histone H3K79 methyltransferase Dot1L is essential for mammalian development and heterochromatin structure. PLoS Genet. 2008;4(9):e1000190.

24. Chen CW, et al. DOT1L inhibits SIRT1-mediated epigenetic silencing to maintain leukemic gene expression in MLL-rearranged leukemia. Nat Med. 2015;21(4):335-343.

25. Deshpande AJ, et al. AF10 regulates progressive H3K79 methylation and HOX gene expression in diverse AML subtypes. Cancer Cell. 2014;26(6):896-908.

26. Okada Y, et al. hDOT1L links histone methylation to leukemogenesis. Cell. 2005;121(2):167-178.

27. Kuntimaddi A, et al. Degree of recruitment of DOT1L to MLL-AF9 defines level of H3K79 diand tri-methylation on target genes and transformation potential. Cell Rep. 2015;11(5):808-820.

28. Bernt KM, et al. MLL-rearranged leukemia is dependent on aberrant H3K79 methylation by DOT1L. Cancer Cell. 2011;20(1):66-78.

29. Nguyen AT, Taranova O, He J, Zhang Y. DOT1L, the H3K79 methyltransferase, is required for MLL-AF9-mediated leukemogenesis. Blood. 2011;117(25):6912-6922.

30. Jo SY, Granowicz EM, Maillard I, Thomas D, Hess JL. Requirement for Dot1l in murine postnatal hematopoiesis and leukemogenesis by MLL translocation. Blood. 2011;117(18):4759-4768.

31. Chen L, et al. Abrogation of MLL-AF10 and CALM-AF10-mediated transformation through genetic inactivation or pharmacological inhibition of the H3K79 methyltransferase Dot1l. Leukemia. 2013;27(4):813-822.

32. Chang MJ, et al. Histone H3 lysine 79 methyltransferase Dot 1 is required for immortalization by MLL oncogenes. Cancer Res. 2010;70(24):10234-10242.

33. Daigle SR, et al. Selective killing of mixed lineage leukemia cells by a potent small-molecule DOT1L inhibitor. Cancer Cell. 2011;20(1):53-65.

34. Daigle SR, et al. Potent inhibition of DOT1L as treatment of MLL-fusion leukemia. Blood. 2013;122(6):1017-1025.

35. Ui A, Nagaura Y, Yasui A. Transcriptional elongation factor ENL phosphorylated by ATM recruits polycomb and switches off transcription for DSB repair. Mol Cell. 2015;58(3):468-482.

36. Leach BI, Kuntimaddi A, Schmidt CR, Cierpicki $\mathrm{T}$, Johnson SA, Bushweller JH. Leukemia fusion target AF9 is an intrinsically disordered transcriptional regulator that recruits multiple partners via coupled folding and binding. Structure. 2013;21(1):176-183.

37. Biswas $\mathrm{D}$, et al. Function of leukemogenic mixed lineage leukemia 1 (MLL) fusion proteins through distinct partner protein complexes. Proc Natl Acad Sci USA. 2011;108(38):15751-15756.

38. Maethner E, et al. MLL-ENL inhibits polycomb repressive complex 1 to achieve efficient transformation of hematopoietic cells. Cell Rep. 2013;3(5):1553-1566.

39. Chen S, et al. The PZP domain of AF10 senses unmodified H3K27 to regulate DOT1Lmediated methylation of H3K79. Mol Cell. 2015;60(2):319-327.

40. Tkachuk DC, Kohler S, Cleary ML. Involvement of a homolog of Drosophila trithorax by 11q23 chromosomal translocations in acute leukemias.
Cell.1992;71(4):691-700.

41. Caldas C, Kim MH, MacGregor A, Cain D, Aparicio $\mathrm{S}$, Wiedemann LM. Isolation and characterization of a pufferfish MLL (mixed lineage leukemia)-like gene (fMll) reveals evolutionary conservation in vertebrate genes related to Drosophila trithorax. Oncogene. 1998;16(25):3233-3241.

42. Shi A, et al. Structural insights into inhibition of the bivalent menin-MLL interaction by small molecules in leukemia. Blood. 2012;120(23):4461-4469.

43. Somervaille TC, et al. Hierarchical maintenance of MLL myeloid leukemia stem cells employs a transcriptional program shared with embryonic rather than adult stem cells. Cell Stem Cell. 2009;4(2):129-140.

44. McGinty RK, Kim J, Chatterjee C, Roeder RG, Muir TW. Chemically ubiquitylated histone H2B stimulates hDot1L-mediated intranucleosomal methylation. Nature. 2008;453(7196):812-816.

45. Wang E, et al. Histone H2B ubiquitin ligase RNF20 is required for MLL-rearranged leukemia. Proc Natl Acad Sci USA. 2013;110(10):3901-3906.

46. Krivtsov AV, et al. H3K79 methylation profiles define murine and human MLL-AF4 leukemias. Cancer Cell. 2008;14(5):355-368.

47. Deshpande AJ, et al. Leukemic transformation by the MLL-AF6 fusion oncogene requires the H3K79 methyltransferase Dot1l. Blood. 2013;121(13):2533-2541.

48. Kühn MW, et al. Targeting chromatin regulators inhibits leukemogenic gene expression in NPM1 mutant leukemia. Cancer Discov. 2016;6(10):1166-1181.

49. Yokoyama A, et al. Leukemia proto-oncoprotein MLL forms a SET1-like histone methyltransferase complex with menin to regulate Hox gene expression. Mol Cell Biol. 2004;24(13):5639-5649.

50. Morita S, Kojima T, Kitamura T. Plat-E: an efficient and stable system for transient packaging of retroviruses. Gene Ther. 2000;7(12):1063-1066.

51. Yokoyama A, Kitabayashi I, Ayton PM, Cleary ML, Ohki M. Leukemia proto-oncoprotein MLL is proteolytically processed into 2 fragments with opposite transcriptional properties. Blood. 2002;100(10):3710-3718.

52. Dull T, et al. A third-generation lentivirus vector with a conditional packaging system. J Virol. 1998;72(11):8463-8471.

53. Daigo K, et al. The proteomic profile of circulating pentraxin 3 (PTX3) complex in sepsis demonstrates the interaction with azurocidin 1 and other components of neutrophil extracellular traps. Mol Cell Proteomics. 2012;11(6):M111.015073. 